\title{
A Computational Analysis of the Relationship between Neuronal and Behavioral Responses to Visual Motion
}

\author{
Michael N. Shadlen, ${ }^{1}$ Kenneth H. Britten, ${ }^{1}$ William T. Newsome, ${ }^{1}$ and J. Anthony Movshon ${ }^{2}$ \\ 1Department of Neurobiology, Stanford University School of Medicine, Stanford, California 94305, and 2Howard Hughes \\ Medical Institute and Center for Neural Science, New York University, New York, New York 10003
}

We have documented previously a close relationship between neuronal activity in the middle temporal visual area (MT or V5) and behavioral judgments of motion (Newsome et al., 1989; Salzman et al., 1990; Britten et al., 1992; Britten et al., 1996). We have now used numerical simulations to try to understand how neural signals in area MT support psychophysical decisions. We developed a model that pools neuronal responses drawn from our physiological data set and compares average responses in different pools to produce psychophysical decisions. The structure of the model allows us to assess the relationship between "neuronal" input signals and simulated psychophysical performance using the same methods we have applied to real experimental data.

We sought to reconcile three experimental observations: psychophysical performance (threshold sensitivity to motion stimuli embedded in noise), a trial-by-trial covariation between the neural response and the monkey's choices, and a modest correlation between pairs of MT neurons in their variable re- sponses to identical visual stimuli. Our results can be most accurately simulated if psychophysical decisions are based on pools of at least 100 weakly correlated sensory neurons. The neurons composing the pools must include a broader range of sensitivities than we encountered in our MT recordings, presumably because of the inclusion of neurons whose optimal stimulus is different from the one being discriminated. Central sources of noise degrade the signal-to-noise ratio of the pooled signal, but this degradation is relatively small compared with the noise typically carried by single cortical neurons. This suggests that our monkeys base near-threshold psychophysical judgments on signals carried by populations of weakly interacting neurons; these populations include many neurons that are not tuned optimally for the particular stimuli being discriminated.

Key words: motion perception; area MT; neural modeling; signal detection; visual psychophysics; sensory coding
How do physiological events in sensory areas of the brain give rise to perceptual performance? To address this fundamental problem, we have drawn from two experimental traditions, sensory neurophysiology and psychophysics. In this we follow Mountcastle and his colleagues, whose work remains a fundamental point of reference for the study of perception (Werner and Mountcastle, 1963; Mountcastle et al., 1969, 1972). By comparing the responses of neurons in somatosensory cortex of the monkey with psychophysical measurements obtained from human and monkey subjects, Mountcastle and his colleagues identified potential cortical substrates for specific tactile sensations. We have extended this approach to analyze neural events underlying the perception of visual motion.

We recorded from directionally selective neurons in the middle temporal visual area (MT) of rhesus monkeys (Allman and Kaas,

\footnotetext{
Received Sept. 11, 1995; revised Nov. 14, 1995; accepted Nov. 20, 1995.

This work was supported by the National Eye Institute (W.T.N., EY 05603; K.H.B., NS07158; J.A.M., EY02017) and by the Howard Hughes Medical Institute (J.A.M., M.N.S.). We thank Peter Földiàk, Stanley Klein, Laurence Maloney, Richard Ulshen, and Brian Wandell for help with mathematics, and Ehud Zohary, Simona Celebrini, Daniel Salzman, and Norma Graham for helpful discussions. We are grateful to H. B. Barlow for suggesting the model described in Appendix 4.

Dr. Shadlen's present address: Department of Physiology and Regional Primate Research Center, University of Washington School of Medicine, P.O. Box 357290 , Seattle, WA 98195-7290.

Dr. Brittcn's present address: Center for Neuroscience, University of California, Davis, CA 95616

Correspondence should be addressed to Michael N. Shadlen, Department of Physiology and Biophysics, and Regional Primate Research Center, University of Washington School of Medicine, P.O. Box 357290, Seattle, WA 98195-7290.

Copyright 1996 Society for Neuroscience $0270-6474 / 96 / 161486-25 \$ 05.00 / 0$
}

1971; Dubner and Zeki, 1971; Van Essen et al., 1981) while the animals discriminated the direction of motion in a stochastic visual display. Our principal innovation was to measure neuronal responses and psychophysical judgments simultancously, under conditions in which the activity of the neuron under study was most likely to contribute to the perceptual judgment made by the monkey. The results provide three important insights concerning the relationship between physiology and psychophysics. First, optimally stimulated MT neurons are, on average, as sensitive to the direction of motion in our stochastic display as is the monkey psychophysically (Britten et al., 1992). Second, there is a subtle but reliable trial-by-trial association between the activity of a single MT neuron and the bchavioral choices made by the monkey. Different presentations of the same motion stimulus do not elicit identical neuronal responses and, near psychophysical threshold, this variable neuronal response appears to be linked to the monkey's choice (Britten et al., 1996). Finally, stochastic response fluctuations are correlated between nearby MT neurons, suggesting that these neurons share a common noise source (Zohary et al., 1994).

In this paper, we attempt to integrate these experimental observations into a model of the process by which sensory signals carried by cortical neurons generate psychophysical performance. The model simulates performance by pooling neuronal responses (based on responses actually recorded from MT neurons) and invoking a simple comparison to produce psychophysical decisions. The structure of the model allows us to assess the relationship between "neuronal" input signals and simulated psychophys- 
ical performance using the same metrics we have applied to real experimental data. Our goal was to establish whether, and under what conditions, the model accurately reproduces the relationships observed in the experimental data, and thereby to gain insight into the neural processes underlying perceptual judgment.

To reconcile simulation results with experimental data, we found it necessary to address four key issues: (1) the number of neurons within the sensory pools underlying performance; (2) the degree of correlated firing among these neurons; (3) the fidelity with which motion signals are encoded by individual neurons within the sensory pools; and (4) the amount of noise that arises after the sensory representation but before the psychophysical decision. Our experimental data-mostly from single neuronsare ambiguous about each of these issues, although multiunit recordings in MT provide an approximate upper bound on the degree of correlated firing, as discussed below. We therefore explored the effects of these four quantities by casting them as free parameters in various simulations.

We find that experimental results can be simulated accurately if psychophysical decisions are based on pools of weakly correlated sensory neurons. In the form of the model that best fits the experimental data, pools are composed of at least 100 neurons but may include many times this number, because model predictions are essentially identical for all larger pools of correlated neurons. The pools must include many neurons that are not optimally tuned to the particular stimuli being discriminated. Central sources of noise degrade the signal-to-noise ratio of the pooled signal, but this degradation is relatively small compared with the noise typically carried by single cortical neurons.

Preliminary accounts of some of this work have been presented previously (Britten et al., 1992; Newsome et al., 1995).

\section{Background}

The simulations in this paper attempt to link physiological and psychophysical data obtained in the context of a specific visual discrimination task. A brief review of the psychophysical paradigm and of our previous experimental results is necessary to appreciate why we adopted this particular model architecture, to enumerate the physiological constraints on the model, and to understand the methods we used to analyze the simulation results (Britten et al., 1992, 1993, 1996; Zohary et al., 1994).

The experimental data were obtained from 254 experiments in four rhesus monkeys (Macaca mulatta). In each experiment, action potentials were recorded from a directionally selective MT neuron while the monkey performed a two-alternative forcedchoice discrimination of motion direction. The monkeys discriminated opposed directions of motion in a set of dynamic random dot patterns that contained a unidirectional motion signal embedded in a field of random motion noise. The strength of the motion signal, which we term the coherence of the display, could be varied from trial to trial by changing the percentage of dots carrying the unidirectional (i.e., "coherent") motion signal.

In the recording experiments, we measured neuronal responses under circumstances in which the activity of the neuron under study was likely to influence the monkey's psychophysical judgments. The psychophysical discriminanda were tailored to activate each neuron optimally. Random dot stimuli were centered within the receptive field of the neuron. The axis of the direction discrimination was set to the preferred-null axis of the neuron (i.e., the direction that yielded best response and its opposite), and the speed of the coherent motion was set to the preferred speed of the neuron. Preferred and null direction stimuli were presented with equal frequency over a range of coherence levels spanning psychophysical threshold. The response of the neuron was considered to be the number of action potentials occurring during each two second presentation of the visual stimulus. In addition to the neuronal data, we recorded the monkey's decision on each trial.

These experiments yielded three salient results that constrain our model. Figure $1 A$ illustrates the finding that the sensitivity of an average MT neuron to directional signals in the stochastic display is equal to the behavioral sensitivity of the monkey. The open circles (dashed curve) are psychometric data depicting the proportion of correct decisions made by the monkey as a function of motion coherence. The closed circles (solid curve) indicate the performance expected of an ideal observer judging the direction of stimulus motion by monitoring the responses of the neuron. Each neuronal dala point was derived from a signal detection analysis of the responses of the neuron to preferred and null direction motion at the appropriate coherence level (see Appendix 1) (Britten et al., 1992). From this analysis, we derive a "neurometric" function that expresses the sensitivity of the neuron to directional signals in a form commensurate with the monkey's behavioral sensitivity measured on the same set of trials. For the experiment illustrated in Figure $1 A$, the MT neuron signaled the direction of stimulus motion with a fidelity comparable with psychophysical performance.

From each of the fitted curves in Figure 1A, we extracted two parameters: a threshold parameter indicating the motion coherence level that supports $82 \%$ correct performance by the monkey, and a slope parameter reflecting the steepness of the relationship between performance and motion coherence. The threshold and slope correspond to the constants $\alpha$ and $\beta$ in the best-fitting cumulative Weibull distribution function (Quick, 1974):

$$
p=1-0.5 \exp \left(-\frac{c}{\alpha}\right)^{\beta}
$$

where $c$ is motion coherence and $p$ is the probability of a correct choice. The similarity of the neuronal and psychophysical data varied widely across the 254 experiments in our study, with some neurons being considerably more and others considerably less sensitive than the monkey. On average, however, the ratio of neuronal to psychophysical threshold was 1.19 and the ratio of neuronal to psychophysical slope was 0.995 , indicating that the near identity of neuronal and psychophysical performance in Figure $1 A$ was typical of the data set as a whole (Britten et al., 1992).

Figure $1 B$ illustrates the second major experimental result that constrains the model (Celebrini and Newsome, 1994; Britten et al., 1996). The figure depicts the responses of an MT neuron to 80 successive presentations of a $0 \%$ coherence stimulus (these trials were interleaved with other, nonzero coherence trials in the actual experiment). Closed circles indicate trials on which the monkey reported seeing the preferred direction of motion, and open circles depict trials on which the monkey reported null direction motion. The frequency histograms at the right were obtained by summing the individual data within horizontal bins. Thus, the solid bars form a distribution of responses observed when the monkey reported the preferred direction, whereas the open bars comprise an analogous distribution for decisions favoring the null direction. Comparison of the two distributions reveals a covariation between the responses of this neuron and the psychophysical judgments of the monkey: decisions favoring the preferred direction predominated when the neuron fired strongly, whereas null 

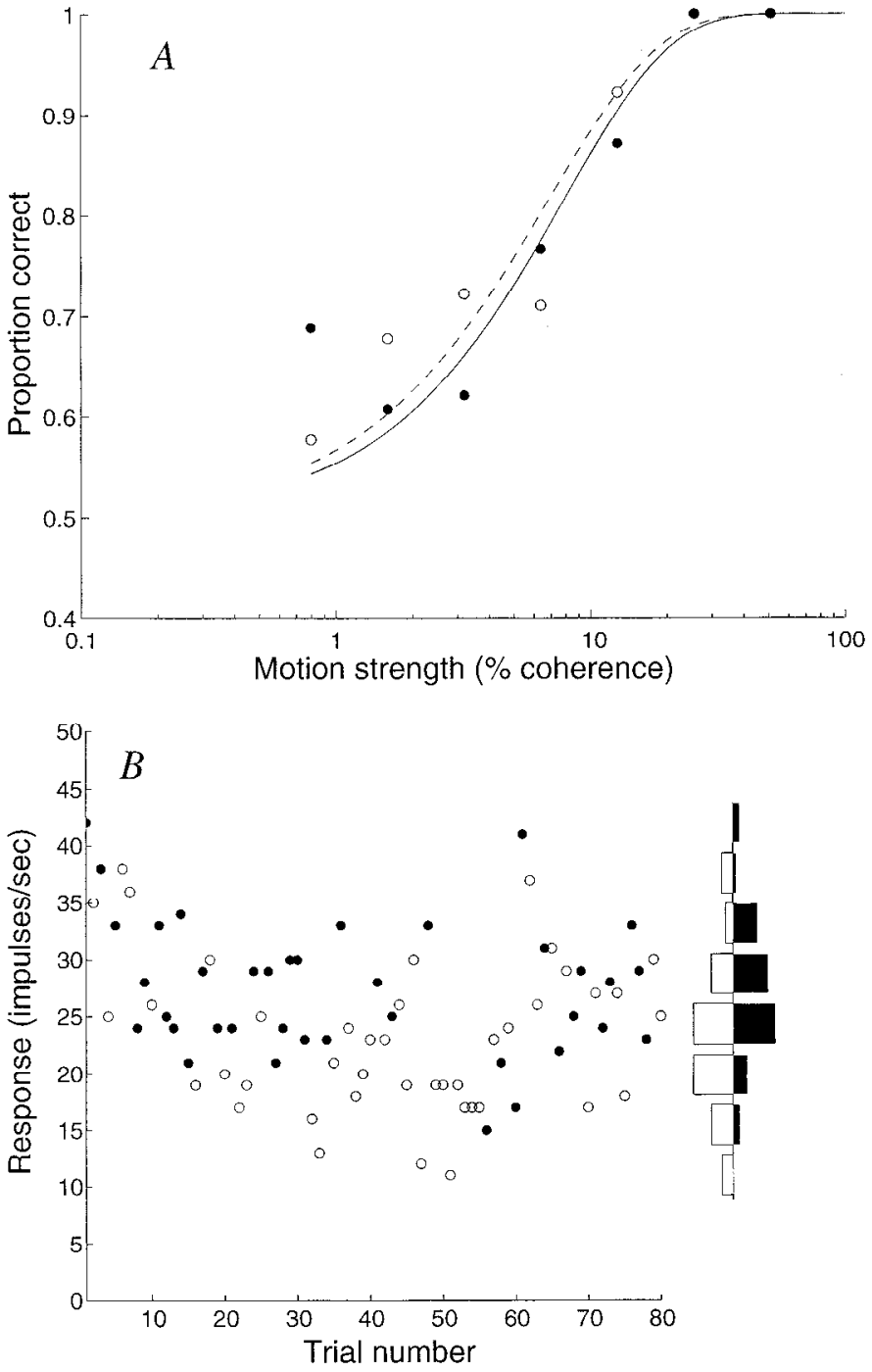

Figure 1. Two experimental observations relating neuronal activity in MT to psychophysical decisions. $A$, Psychometric and neurometric functions collected in one experiment (e065). Open symbols and dashed curve depict the monkey's performance on a two-alternative forced-choice discrimination of motion direction as a function of stimulus strength. The filled symbols and solid curve show the performance that would be achieved by an ideal observer monitoring the spike discharge from the MT neuron. Curves are best-fitting cumulative Weibull functions. In this example, which is representative of the average, the neuron was nearly as sensitive to stimulus motion as the monkey. The stimuli used in this experiment were tailored to the receptive-field preferences of the neuron, matching its preferences for location, size, speed, and direction (preferred and null). $B$, Response from neuron e065 on 80 presentations of the $0 \%$ coherent motion stimulus. Solid and open symbols indicate trials in which the monkey judged motion to be in the preferred and null direction, respectively, of the neuron. The distributions of responses for each type of trial are shown by the histograms (right). The difference between these distributions reveals that on a trial-to-trial basis, there was a weak covariation between the monkey's judgments of motion direction and the variation in neural response. The spike count from this neuron predicted the monkey's choice with $68 \%$ accuracy, a value we refer to as choice probability.

direction decisions were more frequent when the neuron fired weakly. Note that the only difference between the trials included in the two frequency histograms was the monkey's decision; the visual stimulus itself had the same coherence on each trial. In other words, the response variance of this single M'l' neuron could account for a measurable fraction of the variance in psychophys- ical decisions for repeated presentation of a constant subthreshold stimulus.

To quantify the effect in Figure $1 B$, we constructed a receiver operating characteristic (ROC) from the two frequency histograms (Green and Swets, 1966). The normalized area under this curve, which we term the "choice probability," provides a criterion free measure of the discriminability of the two behaviors based on the neural response. The choice probability represents the accuracy with which an ideal observer could predict the monkey's decision from the response of the neuron, given previous knowledge of the distributions in Figure $1 B$ (see Appendix 1). The analysis reflects the association of the responses of the neuron with the behavioral output of the system rather than with the sensory input to the system. A choice probability of 0.5 reflects a randon association between neuronal response and decisions, indicating that the cell provides no basis for predicting the monkey's choices. A value of 1.0 represents perfect predictive power. Across our sample of MT neurons, the mean choice probability was 0.55 , significantly greater than 0.5 (permutation test, $p<$ 0.0005 ; see Britten et al., 1996). For the data set on which most simulations in this paper are based, the value was 0.59 . Thus, small fluctuations in the responses of MT neurons are related to nearthreshold fluctuations in the monkey's decision process.

It is natural to suppose that the brain combines the activity of neurons having similar stimulus preferences. Yet combining activity within a pool of neurons should reduce noise sharply, yielding psychophysical sensitivity substantially better than the sensitivity of individual neurons. Similarly, if the pool were at all large, the response variance of any single neuron in the pool should have negligible impact on the monkey's decisions. Thus, each of our findings suggests that the pools of neurons underlying performance are very small, limiting the beneficial effects of averaging and raising the possibility that noise carried by each neuron would measurably influence the outcome of nearthreshold decisions. Because these notions depend on the noise carried by different neurons being statistically independent, a critical piece of data for resolving this issue is the level of correlated noise among neurons in the sensory pool (Newsome et al., 1989; Britten et al., 1992). To consider an extreme example, trial-to-trial fluctuations in responsiveness to a given stimulus might be perfectly correlated among neurons in the pool. In this case, pooling cannot average out common noise and, therefore, would confer no benefit for psychophysical sensitivity.

Zohary et al. (1994) conducted a multiunit recording study to measure the amount of correlated noise shared between neighboring MT neurons. They recorded simultaneously from two (and on rare occasions three) MT neurons while random dot stimuli were presented within the receptive fields of the neurons. They calculated correlation coefficients between the responses of the two neurons to repeated presentations of each stimulus condition. The correlation coefficients were independent of stimulus condition and therefore were combined across conditions to obtain a single coefficient for each pair of MT neurons. The coefficients varied widely around a mean of 0.12 . For pairs that shared similar direction preferences (separated by $<90^{\circ}$ ), the mean coefficient was 0.19 . Both mean values were significantly greater than zero ( $t$ test, $p<0.0001$ in both cases), and we therefore conclude that MT neurons carry weakly correlated noise during performance of the direction discrimination task.

Modestly correlated noise can fundamentally limit the sensitivity gains accrued by pooling (Johnson et al., 1973; Johnson, 1980b; van Kan et al., 1985; Britten et al., 1992; Gawne and Richmond, 


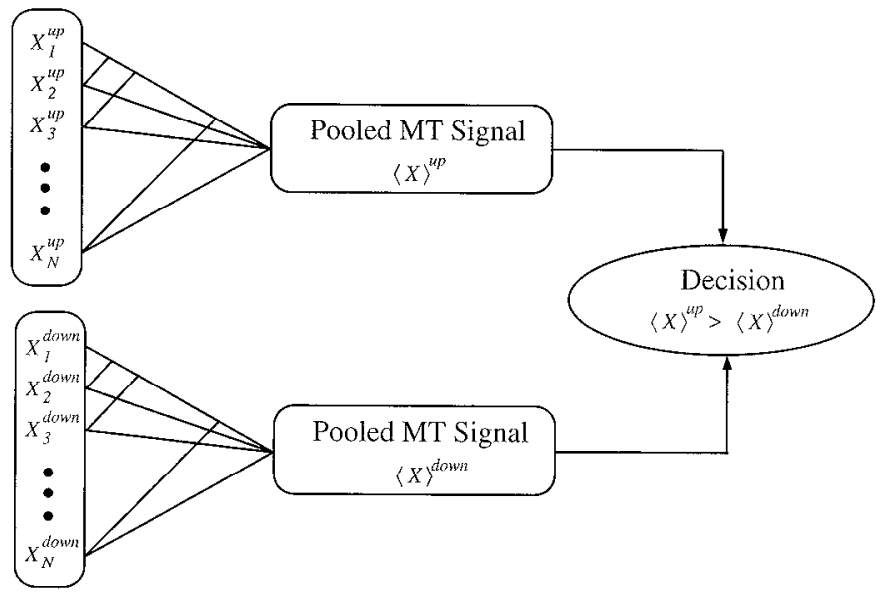

Figure 2. Wc simulatcd psychophysical judgments by comparing neural signals in two pools. On each trial the response from $N$ neurons preferring motion in the direction of stimulus motion, and $N$ neurons preferring motion in the opposite direction are represented by the random variables $X_{I}^{u p} \ldots X_{N}^{u p}$ and $X_{I}^{\text {down }} \ldots X_{N}^{\text {down }}$, respectively. Intersecting lines indicate that these neural responses may be weakly correlated. The responses from each pool are averaged. The larger Pooled MT Signal dictates the Decision. The expected response for each neuron was derived from physiological data.

1993) and can lead, in principle, to a covariation of ncuronal response and psychophysical decision even when the relevant neuronal pools are large (Zohary et al., 1994). We have not tried previously to reconcile quantitatively the experimentally observed correlation level with the key experimental constraints-neuronal sensitivity to the motion signals, the monkeys' psychophysical sensitivity, and the magnitude of the choice probability. The goal of the current study is to accomplish this reconciliation, and thereby gain insight into the nature and composition of the neuronal pools supporting performance and sources of noise intervening between sensory signal and psychophysical decision.

\section{Simulation methods}

\section{Architecture}

To address these issues, we performed a set of computer simulations using Monte Carlo methods. Our calculations were based on a flexible pooling model, outlined in Figure 2, that permitted us to test several hypotheses concerning the link between neuronal responses and psychophysical decisions. For a single iteration of the model, the input was a visual stimulus of a specified coherence and direction, and the output was a single judgment of the direction of stimulus motion. The stinulus elicited responses in two pools of sensory neurons that were modeled after the directionally selective MT neurons recorded in our experiments. In performing the discrimination, we assume that the monkey monitors two pools of neurons with opposed preferred directions. Because neither direction is intrinsically privileged, it seems unlikely that the monkey would monitor neurons selective for only one direction. Rather, decisions are likely to result from a comparison of the evidence in favor of each alternative. Following this logic, our model chose the most likely direction by comparing activity in the two neuronal pools. Rules for pooling responses and forming decisions are indicated below.

\section{Input}

Neuronal responses. Neuronal inputs to the model were simulated from physiological data for the 254 MT neurons described above.
For each neuron in our database, we measured spike counts for a minimum of 10 repetitions per stimulus condition tested (direction and coherence level). The mean spike count thus defines the expected response of each neuron for each stimulus condition. Because response magnitude is a reasonably linear function of stimulus strength for most MT neurons (Britten et al., 1993), we occasionally interpolated between measured response values to generate an expected response to stimulus strengths that were not actually tested in a particular experiment.

To perform a simulation, we randomly selected a pool of $N$ neurons (with replacement) from the MT cells in our database. We then simulated the responses of each neuron to several hundred stimulus presentations, using random dot stimuli covering a range of near-threshold motion strengths. On each simulated "trial," the response of each neuron in the pool was generated as a random pick from a normal distribution whose mean was the expected response for the stimulus presented, and whose variance was 1.5 times the expected response (see below). The object was to remain faithful both to the average responses of real MT neurons and to the trial-to-trial variability in these responses (Snowden et al., 1992; Britten et al., 1993).

Each neuron actually contributed two response values on each trial, one drawn from its distribution of responses to preferred direction motion (pool $X_{I}^{u p} \ldots X_{N}^{u p}$ in Fig. 2) and one drawn from the corresponding distribution for null direction motion (pool $X_{1}^{\text {down }} \ldots X_{N}^{\text {down }}$ ). Up and down are arbitrary designations that allow us to conceptualize a specific experiment, and from here on, we imagine that the preferred direction of every neuron in our data set is upward. 'I'hus, each neuron in the data set doubled as a second neuron having identical response statistics but the opposite direction preference. This procedure instantiates the reasonable assumption that the composition of the two pools is similar in all respects except the preferred direction of motion. For the $0 \%$ coherent motion stimulus, the expected response of any neuron is the same for both pools. The Monte Carlo methods used in generating these responses are described in more detail in Appendix 1.

In simulating neuronal responses, we attempted to mimic the variability observed in our data. The variance of the spike count, measured over multiple repetitions, is nearly proportional to the mean response (Britten et al., 1993). Although this relationship is often described by a power law (Tolhurst et al., 1983; Vogels et al., 1989; Snowden et al., 1992; Britten et al., 1993), the departure from proportionality is negligible (Dean, 1981). Our own data suggest that the variance is $\sim 1.6-2.0$ times the mean (95\% confidence interval for linear regression). Because these physiological data are typically acquired over several tens of minutes to hours, the ratio is likely to be inflated by slow drifts in responsiveness (Dean, 1981; Bradley et al., 1987). Controlling for this effect, Dean (1981) found that response variance was reduced to 1.5-1.7 times the mean response. We adopted the lower value of 1.5 for all simulations in this paper. We performed simulations using larger values (within the observed range) and power law relationships, but these manipulations did not affect our results appreciably. Notably, the variance of the neural response is not attributable to variations in the stochastic motion display because removal of stimulus variance (i.e., presenting the exact same pattern of dots on every trial) does not alter our estimate of variability (Britten et al., 1993).

Number of neurons. The number of neurons, $N$, that provide input to the pools was a free parameter in our early simulations, because one of our major goals was to determine the range of pool 
sizes compatible with our experimental data. Thus, the outcome of our simulations is sometimes plotted as a function of pool size. The number of simulated inputs was typically varied from 1 to 1024. When $N=1$, the pooling model reduces to the simple neuron-antineuron formulation we have used in the past to measure the sensitivities of single neurons (Britten et al., 1992; Celebrini and Newsome, 1994). When $N$ is large, several simulated ncurons may bc modeled on data from the samc ncuron in our database. This allows us to simulate pool sizes larger than our original data set, a technique commonly used in statistical bootstrapping (Efron and Tibshirani, 1993). Because the responses of simulated neurons are stochastic, each "copy" gives rise to its own simulated responses and, thus, each may be regarded as a distinct neuron. Depending on which $N$ neurons are chosen as inputs, the simulated psychophysical data can vary substantially. To minimize the impact of the selection procedure, we repeated each simulation several hundred to several thousand times (depending inversely on $N$ ), randomly selecting a different set of $N$ neurons for each simulation. The results presented in this paper are mean values for all repeated simulations of a particular pool size.

Covariance between neurons. Weak covariance in the activity of sensory neurons can dramatically affect the outcome of a pooling process (Johnson et al., 1973; Zohary et al., 1994). In the present calculations, we modeled corrclated activity among neurons by enforcing a prescribed covariance on their responses. At the beginning of a simulation, when populating the two pools with input neurons, we generated a set of coefficients that described the expected correlation between any member of the pool and each of the other neurons in that pool. For present purposes, we assume no correlation between the two pools (see below). In most instances, the correlation, $r_{i j}$, between any pair of neurons in a pool was a random value between 0 and 0.4 . In practice, this range yielded a mean correlation coefficient of 0.18 , approximating the value measured experimentally (Zohary et al., 1994). Because the measurements of correlated firing in MT were obtained with a single electrode from neighboring MT neurons, the values may be considered as an upper bound to the actual degree of correlation within the sensory pools. More distantly separated neurons are likely to share less common input, leading to less response covariance. We conducted simulations incorporating various corrclation values to determine whether our conclusions depend critically on the precise amount of correlation in the pools. A method for simulating random responses with a desired set of correlations is described in Appendix 1.

Suboptimally stimulated neurons. In our physiological experiments, we always tailored the psychophysical discriminanda to activate optimally the neuron under study. Simulations based solely on such data implicitly assume that optimally tuned neurons are the only ones that influence decisions. If this assumption were flawed, however, such simulations would incorporate an inflated estimate of the average sensitivity of the sensory neurons underlying performance. We therefore modeled the effects of including neurons less sensitive to the stimuli being discriminated, casting the average sensitivity of the input neurons as a free parameter.

To model less sensitive neurons, we randomly assigned to each neuron in the pool a scaling factor between zero and one. We used this factor to modify the expected response of the neuron to motion of any given strength. The procedure had no effect on the responses to $0 \%$ coherence stimuli (random noise) but attenuated any change in spike discharge associated with an increase in stimulus motion strength. For each neuron, we subtracted the response to $0 \%$ coherence from the response to every other stimulus condition, and multiplied these differences by the assigned scaling factor to yield the new expected response function for a "less sensitive neuron." Again, the variance for each stimulus condition was estimated as 1.5 times the new mean. This maneuver retains the realistic response distributions of the original data, while attenuating the sensitivity of the neuron to random dot motion.

Although it is rational that the scaling factors should bc bounded between zero and one, it is not obvious how the factors should be distributed over this interval. We show in Appendix 2, however, that the actual shape of the distribution is irrelevant; simulation results depend on the average sensitivity of neurons in the pool (and their correlation). In our simulations, therefore, we tested the effects of different sets of scaling factors that yielded progressively lower average sensitivity values (i.e., higher neuronal thresholds) within the pool of sensory neurons.

\section{Pooling}

Calculating the pooled signals. Each simulated decision reflects a comparison between the activity of two pools of neurons (labeled $X^{\text {zip }}$ and $X^{\text {down }}$ in Fig. 2). Because we do not know how the brain pools these signals, we explored a number of pooling mechanisms, all of which yielded the same basic pattern of results (Britten et al., 1992). For the present study, therefore, we computed a simple average of the inputs because the pooled signal can then be interpreted intuitively in terms of spike rates.

Pooling noise. The brain cannot compute the averaged (pooled) firing rates with arbitrary precision. To reflect this, we perturbed the pooled average by adding what we call pooling noise. On each trial, the averaged values from up and down pools were not compared directly, but provided the mean values of two Gaussian distributions from which the pooled responses were picked at random. We allowed the variance of the pooling noise to scale proportionally to the pooled mean and therefore express pooling noise as a ratio of variance-to-mean-variance of the zero-mean Gaussian noise source, divided by the pooled mean. Parameterizing pooling noise in this manner helped us develop intuitions about the neurons underlying the pooling stage (see Appendix 3). Conceptually, pooling noise encompasses all sources of variability that would intervene between our calculation of the pooled average and the decision.

Note that for simplicity we performed our initial simulations assuming no pooling noise. Thus, Figures 3 and 4 reflect calculations of precisely averaged response values from MT. Pooling noise is incorporated explicitly in Figure 5 and subsequent analyses.

\section{Output}

Decision rule. Simulated psychophysical decisions were computed simply. The model's "choice" was the direction corresponding to whichever pool of neurons gave a larger mean response. As discussed in the preceding paragraph, the mean values could be corrupted by the addition of pooling noise, but the decision rule remained the same: the larger pooled value won.

On all simulated trials, stimulus motion was upward-that is, in the preferred direction of neurons comprising the upper pool in Figure 2. Thus, the simulated decision was correct when the pooled response of the up pool was larger and incorrect when the response of the down pool was larger. The designations of "direction" and "correct" are arbitrary for the $0 \%$ coherent motion stimulus.

Our approach represents one possible architecture of the deci- 
sion process, even though others might be plausible. Rather than pooling and then comparing, for example, we might consider the reverse: comparing responses between pairs or small groups of neurons and then pooling decisions as one would tally votes in an election. We have explored this and other models, some of which seem implausible from a biological point of view and many of which make obviously erroneous predictions (see Appendix 4 for one example).

Predicted psychophysical performance. Once the parameters for a given simulation were specified, the model generated 500 trials for each of several motion strengths, and simulated decisions were tabulated as the percentage of correct choices as a function of stimulus strength. These data were fitted with a sigmoidal curve like those in Figure $1 A$ (Quick, 1974), and threshold and slope parameters ( $\alpha$ and $\beta$, Eq. 1) were extracted as described for the real experimental data. We repeated the entire process several hundred times, each time populating the pools with a new set of neurons, but otherwise enforcing the same set of assumptions. We considered the geometric mean threshold and average slope from these repetitions to be the prediction of the model for psychophysical performance given the initial set of assumptions. We then varied an assumption (e.g., the number of neurons in the pool) and repeated the process, to explore the effect of each model parameter on simulated psychophysical performance.

We compared the mean thresholds and slopes thus obtained with the mean thresholds and slopes actually produced by our monkeys in our recording experiments.

Predicted choice probability. An attractive feature of the model is that the responses of each input neuron and the psychophysical decision were declared explicitly on each trial. We could therefore compute a choice probability for each simulated neuron, to indicate the degree of trial-to-trial association between simulated neuronal responses and simulated psychophysical decisions. We calculated these choice probabilities in the manner illustrated in Figure $1 B$ for a real MT neuron (see also Appendix 1) (Celebrini and Newsome, 1994; Britten et al., 1996). For every simulation, choice probabilities were computed for each neuron at $0 \%$ coherence.

\section{Comparisons with experimental data}

Our central goal was to identify simulation parameters for which (1) simulated psychophysical thresholds matched the geometric mean thresholds generated by our monkeys during the MT recording experiments, and (2) simulated choice probabilities matched the average choice probabilities measured for our MT neurons. Although we were primarily concerned with comparisons of mean values, we found it helpful on occasion to consider distributions of values as well.

At first glance, it might appear inappropriate to model average psychophysical performance using input from all MT cells that we recorded. After all, the responses of individual MT neurons were measured under a wide variety of psychophysical conditions, because the stimulus parameters were adjusted to match the physiological characteristics of each cell. We checked the validity of our comparison procedure by analyzing a subset of experiments performed under stimulus configurations that resulted in psychophysical thresholds near the population mean (11\% coherence). Neuronal thresholds measured in this subset of experiments (4.0$91.7 \%$ coherence) covered nearly the full range of thresholds observed over the entire database (2.4-91.7\% coherence). We concluded that it is reasonable to model average performance using the full set of data from neuronal recordings.
Variability across subjects

Psychophysical sensitivity, neuronal sensitivity, and choice probability were quite similar for two of our experimental animals (monkeys $\mathrm{E}$ and $\mathrm{K}$ ). Aspects of the data varied, however, in the other two animals (monkeys $\mathrm{J}$ and $\mathrm{W}$ ). Coincidentally, monkeys $\mathrm{E}$ and $\mathrm{K}$ are the two animals for which experimental measurements of correlated activity are available (Zohary et al., 1994). Most of our simulations were therefore based on the 90 neurons recorded from these two monkeys. We did, however, also simulate the data from the other monkcys to identify differences that might account for the variations in the experimental data.

\section{RESULTS}

Each simulation incorporated a set of initial assumptions concerning the size and composition of the pools, the level of correlation among neurons in each pool, and the amount of noise at the pooling stage. Given the set of initial assumptions, each simulation generated two main predictions: psychophysical threshold and the choice probability for each neuron in the pool. Because the assumptions incorporated in the model are really hypotheses about the way calculations are performed by the nervous system, the output of the model was constantly checked against actual experimental observations. We wished to identify model conditions that produced thresholds and choice probabilities similar to the mean values observed in our experiments. We first present the simplest form of the model schematized in Figure 2, in which input neurons were restricted to those we actually recorded from MT and pooling noise was assumed to be absent. The simplest model failed to reproduce the experimental data, and this failure could not be overcome simply by manipulating the number of neurons in the pools. To rectify these problems, in subsequent sections we present simulations that used broader criteria for including neurons in the pools, and added pooling noise.

In Figure 2 we showed the structure of the model and our neuronal notation in a general way. In this section, we will elaborate this model and explore some variations on its general form For this purpose, it is useful to adopt the thumbnail sketch representation shown in Figure $3 A$. The circle on the left represents a pool of MT neurons preferring all possible directions of motion. The shading of elements preferring pure up and pure down motion represents a choice of input neurons restricted to those like the ones we recorded, optimized for the discrimination. In this form, signals from these neurons were simply compared and the decision followed the larger signal.

\section{Pool size and correlation}

Figure $3 B$ depicts the familiar influence of pool size on simulated psychophysical threshold (Johnson et al., 1973, 1979; Britten et al., 1992; Zohary et al., 1994). If each neuron contributes an independent signal to the pooled average (i.e., they share no correlated activity), simulated threshold falls with the square root of pool size, approximately, as illustrated by the solid line in Figure $3 B$. This is a natural consequence of signal averaging, analogous to the improved estimation of the mean with increased sample size. If neuronal activity in the scnsory pools is corrclated, however, the common noise induced by correlation cannot be eliminated by averaging; the improvement in simulated threshold stops at a pool size of $\sim 100$ neurons, as illustrated by the dashed line in Figure $3 B$. The dashed curves in Figure 3 were computed with an average correlation coefficient of 0.18 , the average correlation between MT neurons having similar physiological properties (Zo- 

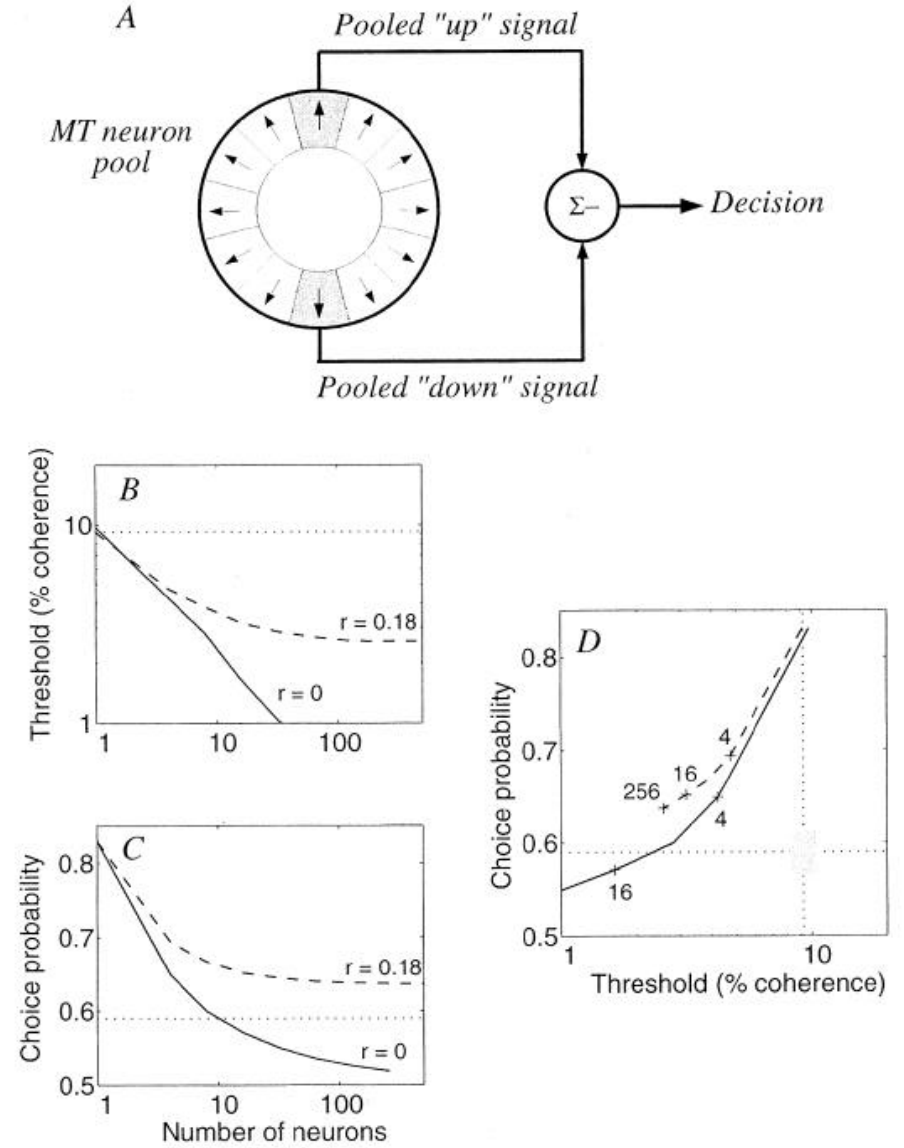

Figure 3. Effect of pool size on threshold and choice probability for independent and weakly correlated neural responses. These simulations used only neurons from our data set and assumed no central pooling noise. $A$, Thumbnail sketch of the model used in these calculations. The Decision reflected a direct comparison of averaged responses from two pools of neurons. The shaded sector indicates that only neurons with appropriate receptive-field properties were included in the pool-that is, neuronal stimulus preferences that matched the direction, speed, and location of the discriminanda. $B$, Predicted Threshold is plotted as a function of the number of neurons in the pools, $N$. When neural responses were assumed to be independent (solid line) the threshold fell roughly with the square root of the number of neurons (slopè $=-0.58$ on $\log$-log scale). When responses were weakly correlated $\left(\bar{r}_{i j}=0.18\right.$, dashed line $)$ threshold fell to an asymptotic value. The horizontal line indicates the mean psychophysical threshold from monkeys E and K. C, Predicted Choice probability is plotted as a function of pool size. When neural responses were assumed to be independent (solid line), the choice probability approached chance $(0.5)$ with increasing pool size. For weakly correlated neurons (dashed line), the average choice probability approached an asymptote of 0.64 . The horizontal dotted line indicates the mean choice probability from the neurons recorded from monkeys E and K. D, Choice probability and predicted Threshold are plotted as a parametric function of pool size. The solid curve was obtained assuming independent neural responses; the dashed curve depicts simulated values when neural responses were weakly correlated. A few values of pool size are indicated to aid in comparison of the two curves. The dashed curve is shorter because predicted values reached an asymptote for $n=50-100$ neurons. In this and subsequent figures, horizontal and vertical dotted lines indicate the mean observed choice probability and threshold (the same values plotted in $A$ and $B$ ); the shaded box represents the mean values $\pm 2 \mathrm{SE}$.

hary et al., 1994). Thus, weak correlation like that observed in MT places fundamental limits on the benefits of signal averaging.

Figure $3 C$ illustrates the influence of pool size on the mean choice probability measured for single simulated input neurons. If each neuron is independent of the others (solid curve), mean choice probability approaches an asymptotic value of 0.5 , indicating only a chance relationship between the monkey's decisions and the variable discharge of any single neuron in the pool. This is not surprising. If the responses of individual neurons fluctuate independently, any one neuron will have minimal impact on the trial-to-trial variation of the pooled average and thus on the decision. Conversely, weak correlation results in a robust choice probability, even for arbitrarily large pool sizes. With an average pairwise correlation coefficient of 0.18 , simulated choice probability approaches an asymptote of 0.64 by $50-100$ neurons (Fig. $3 C$, dashed curve). Weak correlation causes the response of each neuron to covary with the pooled average and hence with the decision (Zohary et al., 1994).

The key question, however, is whether weak correlation can account both for the experimentally observed thresholds and for the choice probabilities. Inspection of Figure $3, B$ and $C$, shows that appropriate values of these two outputs are not obtained for the same values of pool size. Figure $3 D$ illustrates this point in a way we will use a number of times, by means of a parametric plot of simulated choice probability versus simulated threshold for varying pool sizes. This graph shows the same results as in Figure $3, B$ and $C$. The number of neurons is now not shown explicitly but is understood to increase as the curves traverse from high to low values on the threshold and choice probability axes. Again, the solid curve illustrates the results assuming independence of the sensory neurons, whereas the dashed curve depicts the outcome when the neurons in each pool share an average correlation coefficient of 0.18 . The upper right terminus of each curve shows the predicted results when each pool is populated by a single neuron. As pool size increases, both curves sweep down and to the left toward lower choice probabilities and lower thresholds. The dashed curve is shorter than the solid curve because choice probability and threshold reach asymptotic values for large pool sizes.

The dotted lines in Figure $3 D$ represent the mean threshold and mean choice probability actually observed in the 90 experiments from monkeys $\mathrm{E}$ and $\mathrm{K}$ that served as the basis for the simulations. Thus, the intersection of the two dotted lines indicates the locus where simulation agrees perfectly with experiment. The shaded region around the intersection indicates $2 \mathrm{SE}$ on either side of each experimental mean. Clearly, the simulated data do not approach the region that would satisfy the joint constraints of measured thresholds and measured choice probabilities. In essence, thresholds are best modeled by small pools, whereas choice probabilities are best modeled by larger pools. Introducing correlation into the pools helps, particularly for large pool sizes; the lower left terminus of the dashed line (representing large pool sizes) is much closer to the solution locus than the lower left terminus of the solid line. Neither form of the model, however, adequately satisfies the constraints.

We believe the simple form of the model fails because it embodies unrealistic assumptions about the composition of the neuronal pools and about the precision of biological calculations based on the responses in these pools.

\section{Including suboptimally activated neurons in the pools}

We devised a method for simulating the impact on performance of including neurons that were not optimally driven by the stimulus, because we do not know a priori which sensory neurons actually influence the monkeys' decisions. It might be that the monkey heeds signals that arise from neurons whose stimulus preferences do not match the stimuli being discriminated (e.g., 

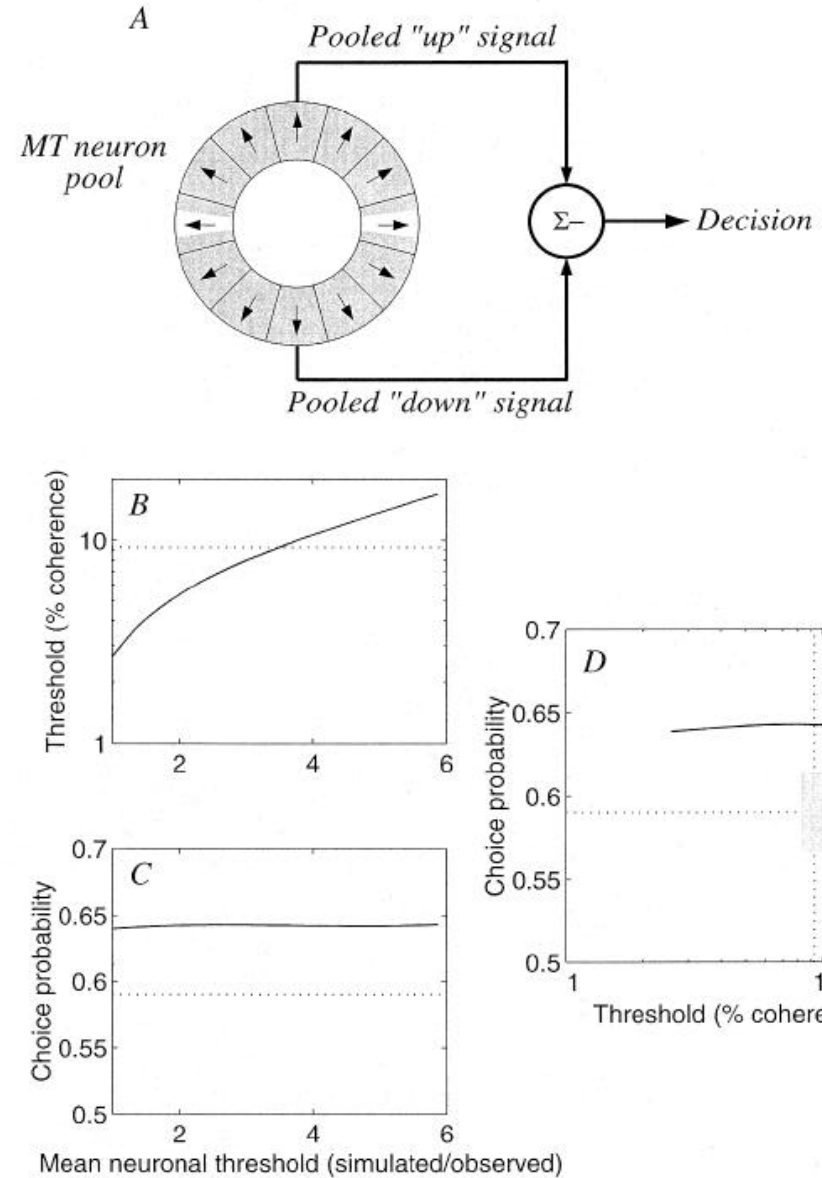

Figure 4. Inclusion of less well activated neurons in the pools affects predicted threshold only. These simulations assumed weak correlation $\left(\bar{r}_{i j}=\right.$ $0.18)$ and asymptotic pool size $(n=128)$; there was no central pooling noise. Dotted lines and box indicate experimentally derived constraints as in Figure 3. A, Thumbnail sketch of the model used to simulate these results. Shaded sectors convey one possible interpretation of the less sensitive neurons, namely, those selective for directions other than upward and downward. The sketch should be taken generically to reflect inclusion of neurons for which receptive-field properties did not match those of the discriminanda. $B$, Predicted psychophysical Threshold is plotted as a function of mean neuronal threshold. The value for neuronal threshold was normalized to the mean obtained using only the neurons in our data set. A value of 1 indicates that only neurons from our data set are included; a value of 2 indicates that the pools contained neurons that were, on average, half as sensitive as those in our data set. $C$, Predicted Choice probability is plotted as a function of Mean neuronal threshold. Inclusion of less well activated neurons in the pool failed to affect the,predicted choice probability. D, Choice probability and predicted Threshold are plotted as a parametric function of mean neuronal threshold. Toward the left, the pools contained only neurons like those in our data set; toward the right, the pools were composed predominantly of relatively inactive neurons.

neurons preferring other directions of motion). Inclusion of such neurons raised the mean neuronal threshold of the pool compared with the mean threshold of the optimally tuned neurons we actually studied. The ratio of these means (simulated neurons to actual MT neurons) provides a convenient way to parameterize the extent to which less sensitive neurons were included in a given simulation. The thumbnail sketch in Figure $4 A$ represents these neurons by widening the range of neuronal preferred directions included in the "up" and "down" pools; note that this illustrative example is only one of several ways in which neurons insensitive to the test stimuli might influence perceptual decisions.
Adding such "insensitive" neurons to the pools is at odds with the notion that pool sizes are small. If decisions are based on a very small number of sensory neurons, the critical neurons should be well tuned to the psychophysical discriminanda; neurons of poor sensitivity should be excluded. (Very small pool sizes appear implausible on other grounds as well, an issue to which we will return in the Discussion.) In all of the following simulations, therefore, we populated each input pool with 128 neurons from the data set of monkeys $\mathrm{E}$ and $\mathrm{K}$, a value that is asymptotically large when the input neurons are weakly correlated (Fig. 3).

Figure 4 shows the effect of including less well activated neurons. An average correlation coefficient of 0.18 was incorporated into the sensory pools to mimic that observed in recordings from MT. Inclusion of these less sensitive neurons in the pools naturally elevated the average single unit threshold and therefore elevated the psychophysical threshold predicted by the model (Fig. 4B). Increasing values on the abscissa reflect progressively more liberal criteria for including neurons in the pools. Toward the left, the pools consist of neurons like the ones we recorded, well matched to the psychophysical task at hand. Toward the right, these matched neurons constitute only a minority of the pool. Figure $4 B$ shows that simulated psychophysical thresholds could be reconciled with observed psychophysical thresholds when the presence of poorly activated neurons raised the mean threshold of the input pools by a factor of 3-4 over that of our sample of MT neurons.

Perhaps less intuitively, Figure $4 C$ shows that inclusion of less well activated neurons had no effect on the simulated choice probabilities, which remained roughly constant over a wide range of pooled sensitivities (solid line). To understand this result, recall that when the input pools are large, a significant choice probability exists only by virtue of correlated firing within the pools. In Figure 3 , for example, the choice probability approaches 0.5 for large pool sizes when the sensory neurons are independent, reflecting a random association between response and decision. When modest levels of correlation were introduced to the pools, however, the asymptotic choice probability increased to a robust value of 0.64 . In essence, any neuron whose response fluctuations are correlated with the pooled signal will exhibit a choice probability; the sensitivity of the neuron is irrelevant. Thus, inclusion of suboptimally driven neurons, although reconciling simulated and experimental thresholds, cannot account for the experimentally observed choice probabilities.

This failure is depicted in the parametric plot of Figure $4 D$. Simulated psychophysical threshold is plotted against simulated choice probability as less sensitive neurons are added to the pool. The threshold ratio (the abscissa in Fig. $4 B, C$ ) is no longer shown explicitly, but is understood to increase as the curve traverses from low to high values on the threshold axis (left to right). The observed thresholds and choice probabilities again are represented by the dotted lines, and the shaded region demarcates $2 \mathrm{SE}$ of each mean observation. The line representing the simulation results lies well outside the shaded region, indicating a failure to satisfy both of our experimental constraints. We now consider solutions of this problem by introducing a new component into the simulations to represent the imprecision of central calculations.

\section{Pooling noise}

The brain must implement pooling operations with real neurons that are themselves sources of variability. We simulated this centrally generated noise by adding a zero-mean noise source to each pooled average response. The variance of the noise distribution quantifies the amount of pooling noise and is conveniently 

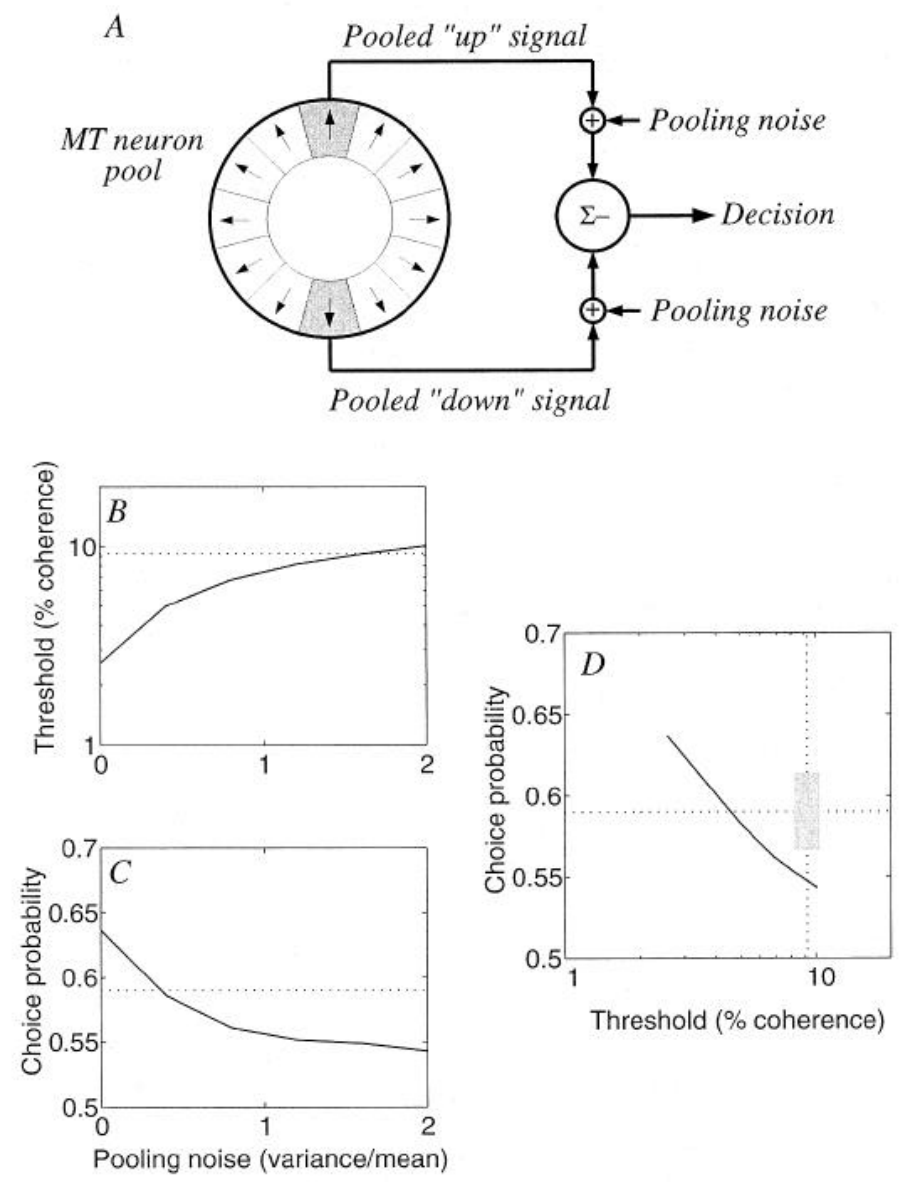

Figure 5. Effect of central pooling noise on predicted threshold and choice probability. These simulations assumed weak correlation $\left(\bar{r}_{i j}=\right.$ $0.18)$ and asymptotic pool size $(N=128)$. Only neurons from our data set were included in the pools. Dotted lines and box indicate experimentally derived constraints as in Figure 3. A, Thumbnail sketch of the model used to simulate these results. Pooled averages were perturbed by a zero-mean Gaussian noise source. The variance of the noise was proportional to the pooled average. B, Predicted psychophysical Threshold is plotted as a function of pooling noise. The latter is parameterized by the ratio: variance of the zero-mean noise source divided by the pooled mean response. Predicted threshold increased as noise was added to the pooled response. $C$, Predicted Choice probability is plotted as a function of Pooling noise. The relationship between neuronal discharge and the simulated decision was attenuated by central pooling noise. D, Choice probability and predicted Threshold are plotted as a parametric function of pooling noise. Notice that different amounts of pooling noise were required to reconcile the choice probability and threshold with experimentally derived values.

described as a fraction of the pooled (average) spike count, or as a variance-to-mean ratio. We now consider the effects of this noise on our model, without including the simulated insensitive neurons we have just considered in Figure 4. Figure $5 A$ shows this form of the model as a thumbnail sketch. The pool of input neurons is again restricted to "pure" up and down neurons, as in Figure $3 A$, but with the addition of pooling noise.

Figure $5 B$ shows the effect of pooling noise on predicted threshold. The simulation incorporated 128 neurons and an average correlation coefficient of 0.18 (i.e., asymptotically large pools). The amount of pooling noise is plotted on the abscissa, with variance-to-mean values ranging from 0 (no pooling noise added) to 2 (substantial pooling noise added). Simulated threshold rose as pooling noise increased, coming into reasonable agreement with experimental observation (dotted line) for variance-to-mean ratios of $1.5-2$.

Unlike adding less sensitive neurons, pooling noise reduced the choice probability (Fig. 5C). To understand this, realize that pooling noise uncouples the decision from the signals in the two pools of sensory neurons. A second source of variation now influences near-threshold decisions, and the new source of variation is unrelated to the signals carried by the input neurons. Thus, pooling noise weakens the association between the sensory signals and the decision. Simulated choice probability could be brought into agreement with observed choice probability (dotted line) when pooling noise attained a variance-to-mean ratio of $0.2-0.4$. Clearly, however, the amount of pooling noise that reconciled simulation and experiment differed substantially for threshold and choice probability.

Figure $5 D$ illustrates this result by plotting simulated choice probability against simulated threshold for different amounts of pooling noise. Pooling noise is low at the upper left end of the curve and increases as the curve tracks downward and rightward. The curve fails to pass through the solution locus representing our experimental results (shaded region), indicating that adding pooling noise alone could not bring the model into conformity with both experimental constraints. Thus, neither noise at the input stage of the model (i.e., less sensitive neurons) nor noise at the pooling stage provided an adequate account of our data.

\section{A successful model}

Figures $4 D$ and $5 D$ suggest that a combination of suboptimally stimulated neurons and pooling noise might account for the experimental data. A thumbnail sketch depicting this kind of model is shown in Figure $6 A$, combining the broad selection rule of Figure $4 A$ with the pooling noise introduced in Figure $5 A$. One recipe for reconciling both observations is to add pooling noise to the basic model, tracking downward and rightward along the curve in Figure $5 D$ until the simulated choice probability agrees with the observed choice probability. Retaining this amount of pooling noise, we then populate the pools with less sensitive neurons, moving our curve rightward (as in Fig. 4D) until simulated psychophysical threshold agrees with observed threshold.

The curves and arrows in Figure $6 B$ illustrate this strategy. The curve on the left shows the results from Figure $5 D$. As we added pooling noise to the model, the curve swept from point A toward higher thresholds and lower choice probabilities. By raising the average threshold of neurons in the pool, we shifted this entire curve directly rightward toward higher thresholds without affecting choice probabilities, causing the curve to intersect the shaded region where both experimental constraints are satisfied. Points $\mathrm{C}$ and D represent simulations with the same amounts of pooling noise as points $\mathrm{A}$ and $\mathrm{B}$, respectively. The $\mathrm{CD}$ curve differs, however, in that the input pools in the model were composed of neurons 2.5 times less sensitive, on average, than those we recorded from MT. The pooling noise associated with points B and $\mathrm{D}$ had a variance-to-mean ratio of 0.3 .

With these particular values, we achieved our goal in the terms we originally established. It is interesting to consider just what these values mean. What does it take to persuade the model to produce the proper outcomes? First, the threshold ratio of 2.5 implies that pooling in the brain is not restricted to optimally tuned neurons, but includes neurons having a broad range of sensitivities. For example, the pools may include many neurons from MT or other visual areas that modulate only weakly along the directional axis of the psychophysical discrimination. This 
A
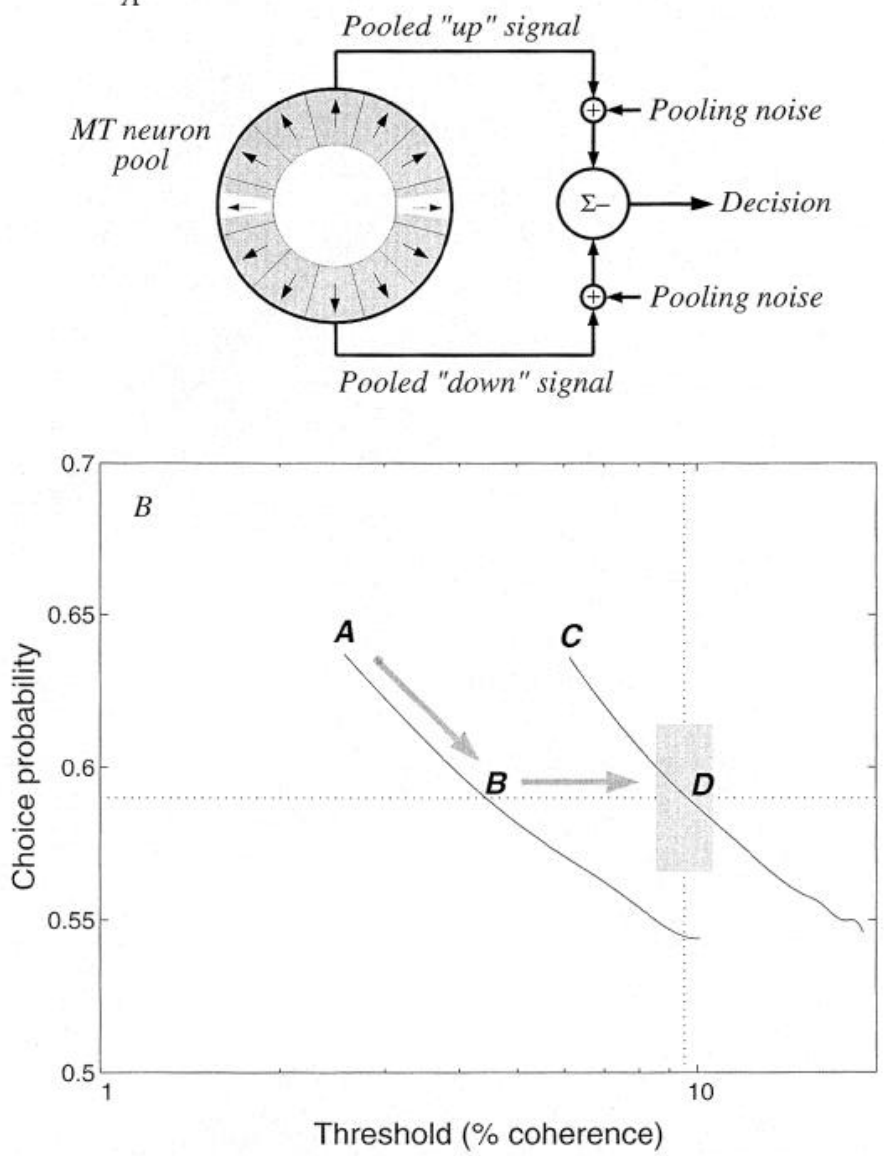

Figure 6. A successful model. These simulations assumed weak correlation $\left(\bar{r}_{i j}=0.18\right)$ and asymptotic pool size $(N=128)$. Dotted lines and box indicate experimentally derived constraints as in Figure 3. A, The model incorporates features of the previous two figures. Pools contained neurons for which preferences did not necessarily match the properties of the discriminanda, and noise was added to the pooled averages. B, Choice probability and predicted Threshold are plotted as a parametric function of pooling noise as in Figure 5. Curve $A B$ was obtained using only neurons from our data set. Curve $C D$ incorporated less sensitive neurons in the pool. The mean neuronal threshold was 2.5 times the mean value from our data set. These curves show how the model parameters can be manipulated to reconcile predicted choice probability and threshold with experimentally derived constraints. Point $A$ marks the predictions using only neurons from our data set and no pooling noise. To reach point $B$, pooling noise was added to achieve the proper choice probability. To reach point $D$, less sensitive neurons were included in the pool.

might mean that neurons selective for directions other than upward and downward influence the monkey's decision, as depicted in Figure $6 A$, or it might imply that neurons with different preferences for speed, or size, or with receptive fields only partly covering the stimulus could play a role. Second, the addition of pooling noise suggests that the brain cannot achieve the degree of noise reduction accomplished by the averaging step in the model, although it comes close. This is not surprising because real neurons, not digital computers, must implement pooling operations in the brain. Notice, however, that the amount of pooling noise incorporated in the model (variance-to-mean ratio $=0.3$ ) is substantially less than the noise typically associated with single cortical neurons (variance-to-mean ratio $\sim 1.5$ ). This is consistent with the idea that psychophysical decisions are based on pooled neuronal signals that have much less variance than that expected of a single cortical neuron (see Appendix 3).

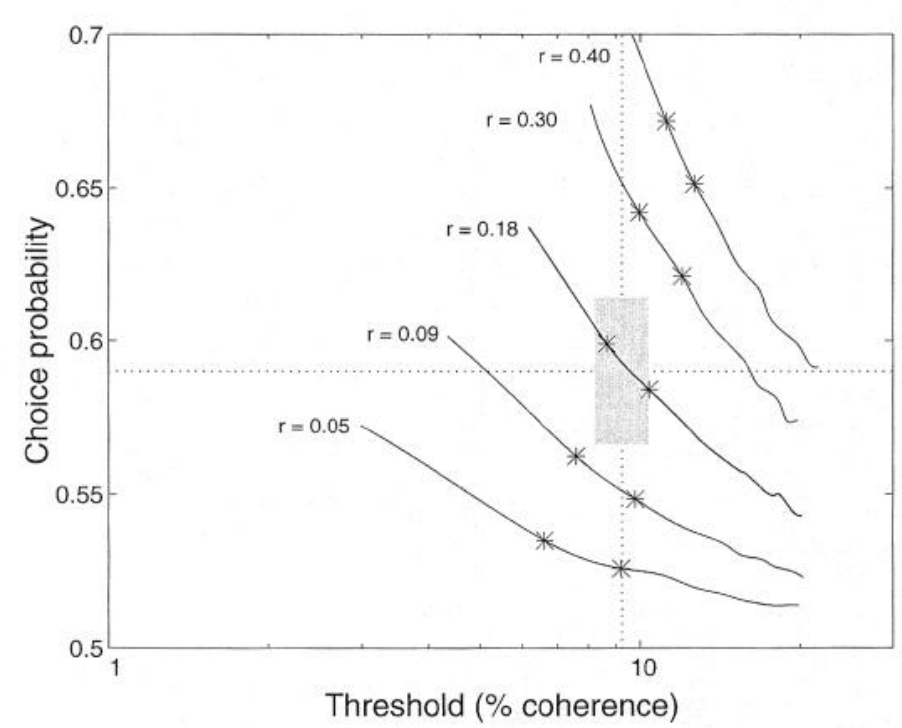

Figure 7. Effect of correlation strength on predicted threshold and choice probability. Choice probability and predicted Threshold are plotted as a parametric function of pooling noise for five values of correlation. The curve representing a mean correlation coefficient of 0.18 corresponds to the successful model shown in Figure 6 (curve $C D$ in that figure). The variance-to-mean ratio of the pooling noise varies from 0 to 2 as the curves traverse from left to right on the graph. Asterisks demarcate variance-tomean ratios of 0.2 and 0.4 . The pools included less sensitive neurons than those recorded from monkeys $\mathrm{E}$ and $\mathrm{K}$ (mean unit threshold $=2.5$ times observed mean). These simulations assumed an asymptotic pool size $(N=$ 128). Dotted lines and box indicate experimentally derived constraints, as above.

The model giving rise to the data in Figure $6 B$ realizes our primary goal of reconciling experimentally observed neuronal sensitivities, choice probabilities, and psychophysical thresholds. The model suggests that perceptual decisions are based on pools of weakly correlated neurons, including many that are not tuned optimally for the particular stimuli being discriminated. Both psychophysical sensitivity and choice probability are degraded modestly by centrally generated sources of noise. We used this basic form of the model to explore several related questions of interest. How are our results affected by changes in the level of correlated firing or, more generally, how do the various model parameters interact? Does the model accurately predict the measured slope of the psychometric function? Can the model account for an experimentally observed correlation between neuronal sensitivity and choice probability? How well does the model accommodate differences among the data sets of individual monkeys?

\section{Dependence on the level of correlated firing}

Of the main observations we seek to reconcile, the degree of correlated firing between neurons is the least determined. This is because the technique of recording adjacent neurons through a single electrode may overestimate the degree of correlation among neurons within the pools. For this reason, we explored the effect of variation in the level of correlated firing on the behavior of the model.

We generated predictions for threshold and choice probability as a function of pooling noise for five different levels of correlated firing (Fig. 7). After the results developed in preceding sections, we populated the pools with neurons 2.5 times less sensitive, on average, than the ones we recorded. The parametric curves sweep from the upper left to the lower right as pooling noise is added to 
the model. For comparison, asterisks demarcate the interval on each curve representing pooling noise with variance-to-mean ratios between 0.2 and 0.4 , the range used in the successful analysis documented in Figure 6.

As indicated previously, simulated and experimental data can agree nicely when the average pairwise correlation within the sensory pools is 0.18 (middle curve). Figure 7 also shows that small changes in the average correlation coefficient led to substantial shifts in the predictions of the model. Notice that the curves representing correlation coefficients of 0.09 and 0.3 miss the shaded solution locus. These simulations could be brought into agreement with experimental observations by adjusting the average sensitivity of the pool, thereby shifting the curves horizontally (compare Fig. 6). Reconciliation was difficult, however, if the pairwise correlation was substantially higher or lower than these values. With an average correlation coefficient of 0.05 , for example, the curve representing the simulated results (Fig. 7, bottom curve) passes below the shaded solution locus. Threshold and choice probability cannot be reconciled simultaneously unless we populate the input pools even more densely with suboptimally activated neurons, moving the lower curve directly rightward on the graph. For this strategy to work, however, we would have to assume a complete absence of pooling noise, which we believe to be implausible (see Appendix 3). For average correlations of $<0.05$, the model failed for any combination of suboptimally activated neurons and pooling noise.

The model is no more tolerant of high correlation coefficients. The top curve in Figure 7, incorporating a mean pairwise correlation of 0.4 , could be forced through the solution locus only if the pools were populated purely with optimally stimulated neurons, shifting the entire curve leftward. For this model to succeed, in fact, the pools had to be populated from a very select group of the most sensitive neurons we recorded. Even then, only the rightmost points on the curve intersected the shaded region, and these points reflected large amounts of pooling noise, with variance-tomean ratios exceeding 1.5. Although such values may not be impossible, we will argue in Appendix 3 that they are unlikely.

The results in Figure 7 provide a useful perspective on our modeling effort. Perhaps most important, they show that the number of free parameters in the model did not guarantee success; plausible solutions depend critically on the degree of pairwise correlation within the sensory pools. Put another way, the model predicts that the average correlation within the pools should lie between 0.1 and 0.3 , a range that corresponds well with the correlation values measured in monkeys $\mathrm{E}$ and $\mathrm{K}$ (Zohary et al., 1994).

\section{Summary of interactions among model parameters}

We have considered several factors that affect the relationship between single-cell discharge and psychophysical decisions: pool size, single-unit sensitivity, correlation, and pooling noise. Figure 8 illustrates qualitatively how these factors influence the model's estimates of psychophysical threshold and choice probability.

Our simplest pooling model, containing only optimally tuned neurons and lacking pooling noise, overestimated the choice probability and underestimated psychophysical threshold, as illustrated by the asterisk. This asterisk corresponds to the upper left point of the curves in Figures $4 D$ and $5 D$. Each arrow in Figure 8 indicates the effect of changing a model parameter. For example, if the pools include less sensitive neurons, then the estimate for threshold rises while choice probability is unaffected. If noise intervenes between MT and the formation of a decision, threshold

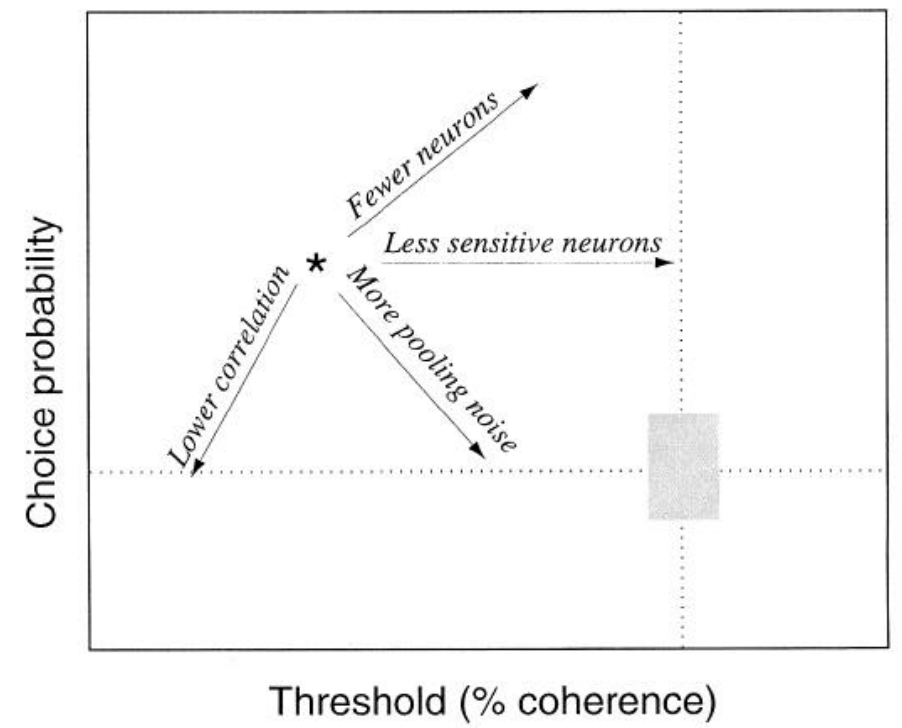

Figure 8. Effect of each of the model parameters on predicted choice probability and threshold. Using experimentally derived measures of neural response and correlation, the pooling model predicted a psychophysical Threshold that is too low and a Choice probability that is too high (asterisk). These estimates pertain to pool size of $>50-100$ neurons. Effects of each manipulation are indicated qualitatively by the direction of the associated arrow. From these heuristics it is possible to predict the qualitative effects of various combinations of parameters. Dotted lines and box indicate experimentally derived constraints, as above.

rises and choice probability falls. If correlation between sensory neurons is weaker, then choice probability becomes smaller while threshold becomes even lower (sensitivity improves). Finally, reducing the number of neurons in the pools would raise the choice probability and increase threshold, whereas larger pools would have negligible affect unless this manipulation were accompanied by a change in average correlation or unit sensitivity.

Figure 8 shows how these factors interact. For example, a slightly lower correlation would necessitate less pooling noise to drive the asterisk into the solution locus, but would require that less sensitive neurons comprise the pools. Similarly, if less sensitive neurons were more weakly correlated with the pool, their impact on sensitivity might be offset partially by a lower average correlation, and choice probability would be lower (see Appendix 2). Although derived from an analysis of motion signals in MT, these heuristics are applicable, in principle, to many neural circuits subserving near-threshold psychophysical decisions (Johnson, 1980b).

\section{Shape of the psychometric function}

We have so far compared the monkey's performance with an estimate of threshold sensitivity. Recall, however, that the monkey's behavior is described by a psychometric function, like the one in Figure $1 A$, which has both a threshold and a slope. The threshold, used extensively in our previous analyses, is the stimulus strength supporting $82 \%$ correct choices. The slope ( $\beta$ in Eq. 1) describes the rate of change in performance with changes in motion strength. An account of the monkey's psychophysical performance should explain both the slope and threshold of the observed psychometric function.

The average slope of the psychometric function for our monkeys was 1.20 (for $\mathrm{E}$ and $\mathrm{K}$, it was 1.23 ); human observers have similar psychometric function slopes on this task (Downing and 


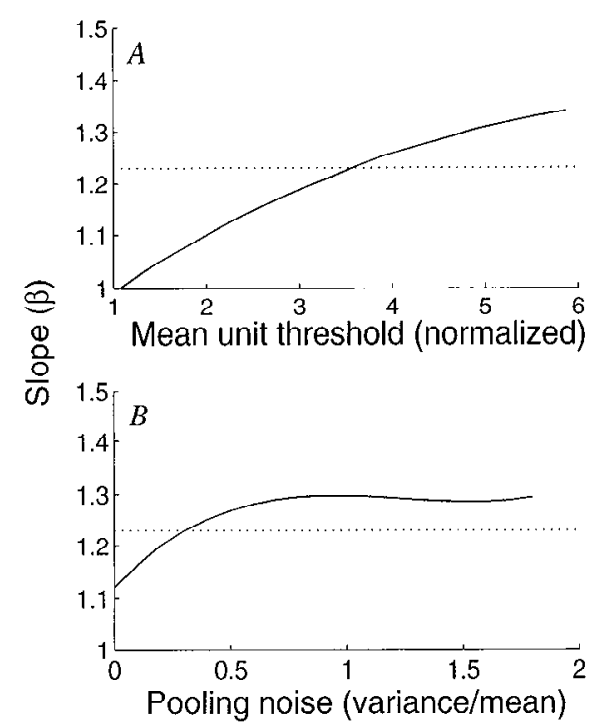

Figure 9. Predicted slope of the psychometric function. The observed mean slope from monkeys $\mathrm{E}$ and $\mathrm{K}$ is indicated by the horizontal dotted line. A, The slope of the PMF is plotted as a function of Mean unit threshold (normalized to the mean of neurons selected from our data set). These simulations used asymptotically large pool size $(N=128)$, weak correlation $\left(\bar{r}_{i j}\right)$, but no pooling noise. A decrease in the sensitivity of the pool was associated with a modest rise in the slope of the PMF. $B$, The slope of the PMF is plotted as a function of increasing Pooling noise. These simulations assumed the same parameters as in $A$, except the mean unit threshold was 2.5 times the mean of neurons selected from our data set.

Movshon, 1989). All of the simulations summarized thus far produced estimates of slope between 1.0 and 1.4. Because of the uncertainty associated with estimation of these slopes (Nachmias, 1980), it may be wise to regard any of these results as compatible with the data. Nevertheless, we observed an interesting effect on slope when adding the two noise sources to the pooling calculations. These effects are summarized in Figure 9. Using asymptotically large pools of weakly correlated neurons recorded from monkeys $\mathrm{E}$ and $\mathrm{K}$, we obtained a mean slope of 1 . By including less sensitive neurons in the pool (Fig. 9A), the slope increased to a maximum value of 1.34 . The simulations used to produce Figure $9 A$ were the same as those used for Figure 4. The abscissa represents the average single-unit threshold normalized to the neurons in our database. When the mean threshold was 2.5 times greater than the neurons in our database, the slope was 1.10-1.15.

Adding pooling noise to the model also modestly increased the slope of the psychometric function, as shown in Figure 9B. To generate this plot, we included less sensitive neurons in the pools so that the average neuronal threshold was 2.5 times greater than the neurons in our sample (same simulations as Fig. 6, curve $C D$ ). Thus, with no pooling noise, the curve intersected the ordinate at $\beta=1$.1. As pooling noise was added to the model, the slope increased to a value of 1.30. It is striking that the model generated a slope of 1.23 (the mean value for monkeys $E$ and $K$ ) when the pooling noise had a variance-to-mean ratio of 0.3 . This is the same combination of pooling noise and the same mixture of neuronal preferences that we found compatible with the observed threshold and choice probability. Although this is gratifying, we recognize that these effects are small, and we are disinclined to exclude any range of model parameters on the basis of this analysis. We have analyzed other classes of models, however, that make more extreme predictions for the slope parameter (Britten et al., 1992), and we will consider one such model in Appendix 4.

\section{Choice probability is greater for more sensitive neurons}

We have described elsewhere an inverse relationship between neuronal threshold and choice probability in both MT and the medial superior temporal visual area (MST): the responses of low-threshold neurons covary more strongly with the decision than do the responses of high-threshold neurons (Celebrini and Newsome, 1994; Britten et al., 1996). In the simulations presented so far, however, the value of choice probability was uniform across the pools of input neurons. We explored two ways to incorporate the heterogeneity of choice probability into the model, taking the successful model depicted in Figure 6 as our starting point.

The inverse relationship between threshold and choice probability suggests that sensitive neurons influence the monkey's decisions more strongly. More sensitive neurons might be connected to the pooling stage with greater weight, thereby influencing the decision stage more effectively. We incorporated this hypothesis into the model by assigning greater weight to the responses of sensitive neurons in the pooling process, basing decisions on suitably weighted averages of activity in the two pools. To our surprise, we could not mimic the experimental observation in this way, although we tried a number of different strategies for assigning weights. We realized that these efforts were frustrated by the correlation of activity within the pools. Recall from previous sections that if the neuron pools are large, choice probability results only from correlated activity within the sensory pools. For example, correlated responsiveness preserved significant choice probabilities even when very poorly activated neurons were incorporated in the sensory pools (Fig. 4). These simulations, therefore, suggest a rather counterintuitive property of MT neurons: if the responses of a neuron covary with the responses of other members of the pool, the responses of that neuron will covary weakly with the decision regardless of the weight assigned to the neuron. Even if a neuron has zcro wcight, its activity will show a choice probability if its response is correlated with that of neurons with nonzero weight.

This insight led us to try to account for the observation that different neurons have different choice probabilities by varying the structure of the correlations among ncurons in the pool. If choice probability is determined by correlation irrespective of weight, choice probability should be changed by varying the pairwise correlation of neurons within the pool. Neurons that are better correlated with the rest of the pool should covary more strongly with the pooled average and hence with decisions, and thus have higher choice probabilities. This hypothesis can be incorporated into the model by assigning an average correlation coefficient to each neuron according to its sensitivity.

We used a variant of the asymptotic model developed in preccding scctions. As bcforc, wc constructcd pools of 128 cells from neurons sampled from monkeys $\mathrm{E}$ and $\mathrm{K}$. The model included less sensitive neurons (average threshold elevated by a factor of 2.5) as in Figures 6 and 7, and pooling noise with a variance-to-mean ratio of 0.3 . Neuronal responses were pooled by simple averaging, but scnsitive neurons were better correlated with other neurons in the pool. We created a gradation in the mean correlation coefficient between a neuron and all others in the pool so that the most sensitive neurons received the highest coefficients, and so on. The most sensitive neuron in the pool had an average correlation coefficient of 0.3 with the other members of the pool, and the least sensitive neuron had an average coefficient of $\sim 0.03$. This procedure yielded a mean correlation coefficient of 0.1 for the popula- 


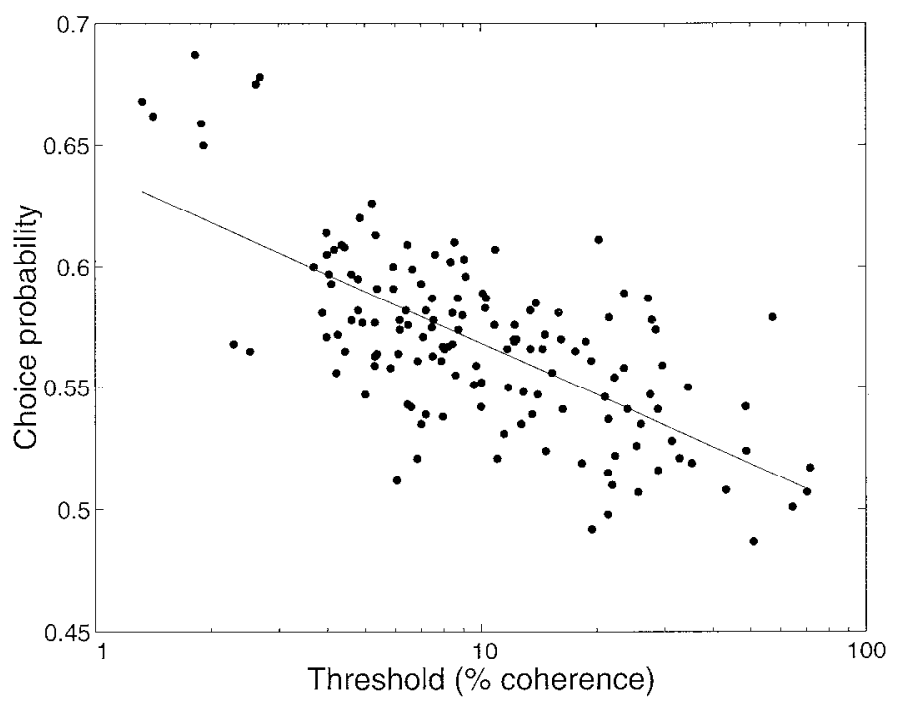

Figure 10. An inverse relationship between neuronal Threshold and Choice probability. In these simulations, neurons were assigned correlation coefficients according to their relative sensitivity: the more sensitive neurons in the pool were assigned higher correlation coefficients with other members. Although the pools were composed of neurons representing a broad range of sensitivity, only those resembling the units in our data set are shown (scaling factor was $>0.9$ ).

tion of neurons at large and 0.16 for the subset of more sensitive neurons that were scaled by $<10 \%$. We considered this latter group to be representative of the neurons actually recorded from MT. For these neurons, the mean choice probability and predicted psychophysical threshold were 0.57 and $8.9 \%$ coherence, respectively, falling within the solution locus (shaded box in Figs. 3-6). Our methods for assigning these highly structured correlation coefficients and performing the calculations are described in greater detail in Appendix 1.

Figure 10 illustrates the outcome of 10 simulations based on this model. The scatter plot shows the relationship between simulated choice probability and neuronal threshold for 146 simulated neurons, revealing a significant inverse relationship between the two parameters. Sensitive neurons appear more closely linked to psychophysical decisions, even though the responses of these neurons were not given additional weight in the pooling process. Their disproportionate influence is attributable solely to stronger covariation with other neurons in the pool, hence with the pooled average and with the decision. Note that only the minimally attenuated neurons are shown in this plot, representing the neurons that we encountered in our experiments. The less sensitive neurons in general have high thresholds and choice probabilities near 0.5 .

The relationship in Figure 10 matches that observed in our experimental data (Celebrini and Newsome, 1994; Britten et al., 1996), and the experimental effect, therefore, may be attributable to a link between neuronal sensitivity and effective connectivity with other neurons in the pool. This hypothesis is supported by physiological evidence that the noise carried by neurons with similar properties is better correlated than that carried by neurons with dissimilar properties (Zohary et al., 1994). If the most sensitive neurons in a pool are those best tuned to the discriminanda, their responses will covary most strongly, presumably because of greater common input.

I'he most important insight from these simulations is that variation in choice probability can arise from variation in the average correlation of each neuron with other neurons in the pool. Unfortunately, we can only speculate on the exact pattern of correlation between all pairs of pooled neurons in the brain. The simulations suggest that correlation coefficients are not randomly related to neuronal sensitivity, but the highly structured set of correlations used in the simulations of Figure 10 seems artificial. A less rigidly constrained covariance structure might be more plausible (see Appendix 1 for additional details).

\section{Individual monkeys}

The simulations presented thus far were based on combined data from two of our four monkeys, E and K. As summarized in Table 1 , these monkeys had similar psychophysical thresholds, singleunit thresholds, and choice probabilities. In addition, our simulations are on firmer ground with these data because our measurements of pairwise correlation were actually made in these two monkeys (7ohary et al., 1994). A third monkey, W, differed from monkeys $\mathrm{E}$ and $\mathrm{K}$ in relatively minor ways, but the fourth monkey, $\mathrm{J}$, differed more dramatically. Can we modify the pooling model to account for the data from monkeys $\mathrm{W}$ and $\mathrm{J}$ ?

Treating the neurons from each animal as independent data sets, we attempted to model the psychophysical threshold and choice probability measured for each animal. Figure 11 depicts the results. As in previous figures, the shaded areas depict solution loci defined by the mean of the experimental observations $\pm 2 \mathrm{SE}$. In each panel, the solid curve illustrates simulated results assuming a mean pairwise correlation of 0.18 and inclusion of suboptimally activated neurons at the level derived above in the combined analysis of monkeys $\mathrm{E}$ and $\mathrm{K}$. Not surprisingly, these parameters worked well for monkeys $\mathrm{E}$ and $\mathrm{K}$ considered independently. A small difference in neuronal sensitivity between the two monkeys compensated for the small difference in psychophysical sensitivity apparent in Figure 11. For monkeys W and I, however, the parameters derived from analysis of monkeys $E$ and $\mathrm{K}$ do not account for the observed data.

Monkey $\mathrm{W}$ differed from monkeys $\mathrm{E}$ and $\mathrm{K}$ in that its neurons were slightly less sensitive and, more important, its average choice probability was substantially lower. Thus, the solid curve in Figure 11 misses the solution locus. There are two ways to remedy this. First, restricting the pools to more sensitive neurons-like the ones we recorded-would shift the solid curve leftward, bringing the lower right end of the curve close to the solution locus. This would imply that monkey $W$ polled a more select subset of neurons, but degraded the pooled signals substantially with pooling noise. The amount of pooling noise necessary to achieve this remedy appears implausible, however, because it exceeds the variability expceted from a single ncuron that would represent the pooled response. We can simulate monkey W's data most effectively by incorporating weaker pairwise correlation into the sensory pools. With an average pairwise correlation of 0.09 , the model reconciled psychophysical threshold and choice probability (Fig. 11, dashed curve). This manipulation is consistent with the intuition developed above that the choice probability reflects correlated responses in the sensory pool; smaller choice probabilities should reflect weaker correlation. Interestingly, the amount of pooling noise that best explained the data of monkey $\mathrm{W}$ is similar to the amount that worked for monkeys $\mathrm{E}$ and $\mathrm{K}$, if the average pairwise correlation is allowed to drop to 0.09 (see Table 1).

We adopted a more substantial change in parameters to simulate the data from monkey $\mathbf{J}$ (Fig. 11, bottom right; Table 1). This monkey is an outlier in several respects because its psychophysical 
Table 1. Comparison of experimental data and optimal simulation parameters for four monkeys

\begin{tabular}{|c|c|c|c|c|c|c|c|c|}
\hline \multirow[b]{2}{*}{ Monkey } & \multicolumn{2}{|l|}{ Neuronal } & \multicolumn{2}{|l|}{ Behavioral } & \multirow[b]{2}{*}{$\begin{array}{l}\text { Choice } \\
\text { probability }\end{array}$} & \multicolumn{3}{|c|}{ Simulation parameter } \\
\hline & $\begin{array}{l}\text { Threshold } \\
\text { (\% coherence) }\end{array}$ & Slope & $\begin{array}{l}\text { Threshold } \\
\text { (\% coherence) }\end{array}$ & Slope & & $\begin{array}{l}\text { Average } \\
\text { correlation }\end{array}$ & $\begin{array}{l}\text { Mean unit threshold } \\
\text { (simulated/observed) }\end{array}$ & $\begin{array}{l}\text { Pooling noise } \\
\text { (variance/mean) }\end{array}$ \\
\hline $\mathrm{E}$ & 17.2 & 1.06 & 11.3 & 1.13 & 0.587 & 0.18 & 2.8 & 0.4 \\
\hline $\mathrm{K}$ & 11.7 & 1.25 & 7.7 & 1.38 & 0.593 & 0.18 & 2.1 & 0.2 \\
\hline W & 15.9 & 1.40 & 8.2 & 1.14 & 0.539 & 0.09 & 2.3 & 0.3 \\
\hline $\mathrm{J}$ & 29.1 & 1.52 & 20.8 & 1.21 & 0.510 & 0.05 & 2.3 & 1.0 \\
\hline
\end{tabular}

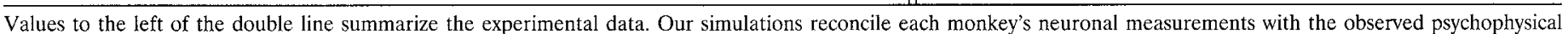

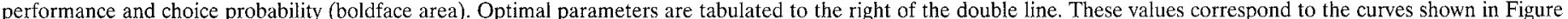

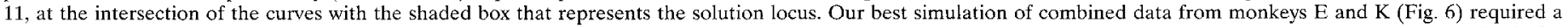

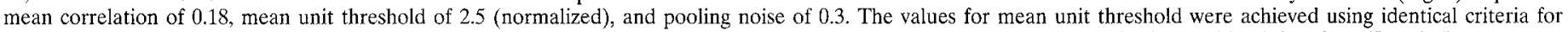
inclusion of suboptimally stimulated neurons (i.e., the same range of response scaling factors that produced a value of 2.5 in the combined data from $\mathrm{E}$ and $\mathrm{K}$ ).

and neuronal thresholds were substantially higher than the other monkcys and its choicc probability was substantially lower (Britten et al., 1996). Interestingly, monkey J was the only animal whose single neuron thresholds were lower, on average, than psychophysical threshold (see Fig. $9 A$ in Britten et al., 1992). In other words, the data from this monkey suggest that the monkey benefited the least from pooling given the sensitivity of its neurons.

These two trends-less benefit from pooling and lower choice probabilities - are at odds with each other according to the logic of our model. We expect choice probabilities to be weak or absent if pairwise correlation in the pools is exceedingly weak. But low correlation should lead to more effective pooling because response covariance is a major obstacle to noise reduction through averaging. A possible solution is to assume that any gains derived from low correlation are offset by high pooling noise, as illustrated by the dashed curve in Figure 11 (bottom right). This curve passes through the solution space when the pooling noise is -1.0 . Recall that the value of pooling noise in our successful model was $\sim 0.3$, consistent with the idea that psychophysical decisions are based on a pooled neuronal signal whose variability was much less than that expected of single neurons. The value needed to fit the data from monkey J, however, implies that the variability of the signal supporting decisions would approach that expected of a single neuron. It seems unreasonable to posit that the pooled neuromal signal is as variable as the single neuron signals that contribute to it. We believe that this solution is different in spirit from the model we have developed, in which pooling by groups of neurons is a key element. We address these issues in more detail in Appendix 3.

Table 1 lists the parameter values that satisfied the constraints derived from experimental observations for each of the four monkeys. These values correspond to the intersection of the curves shown in Figure 11 with the shaded solution loci (solid curves for monkeys $\mathrm{E}$ and $\mathrm{K}$; dashed curves for monkeys $\mathrm{W}$ and $\mathrm{J}$ ). Except for monkey $\mathrm{J}$, model parameters are remarkably similar. In round numbers, the model suggests an average pairwise correlation of $0.1-0.2$, a mean neuronal threshold that is 2.5 times the value obtained for optimally driven neurons, and pooling noise that is $\sim 20 \%$ of the response variance commonly measured in single cortical neurons.

\section{DISCUSSION}

The goal of this study was to gain insight into the relationship between neuronal activity in the cerebral cortex and psychophysical performance on a direction discrimination task. To this end, we developed a quantitative model linking the responses of neu- rons in extrastriate area MT to psychophysical performance measurcd during the physiological recordings. The model was constrained by several experimental observations: (1) the responses of MT neurons to motion signals in the visual displays (Britten et al., 1993); (2) the psychophysical sensitivity of the monkeys to the same motion signals (Newsome et al., 1989; Britten et al., 1992); (3) the shape of the psychometric function (i.e., the rate of improvement in performance with increasing stimulus motion strength) (Newsome et al., 1989; Britten et al., 1992); (4) the trial-to-trial covariation between neuronal responses and psychophysical decisions made by the monkcy (i.c., choicc probability) (Celebrini and Newsome, 1994; Newsome et al., 1995; Britten et al., 1996); and (5) weak correlation among pairs of MT neurons in their response to repetitions of identical stimuli (Zohary et al., 1994). Free parameters in the model included the number and average sensitivity of neurons in the sensory pools and the amount of noise introduced at the pooling stage. The model provided an acceptable account of the various experimental data over a restricted range of conditions.

Together, our experimental and theoretical results suggest several conclusions concerning the relationship of neuronal activity to performance. Psychophysical decisions are hased on a comparison of signals from pools of weakly correlated neurons. The pools are probably composed of at least 100 neurons but may include many times this number because model predictions are essentially identical for all larger pools of correlated neurons. Neurons contributing to the pools are likely to include many that are not optimally tuned to the particular motion stimuli being discriminated. Central sources of noise are likely to degrade the pooled signal, but this degradation appears small in comparison with response fluctuations typically measured in single cortical neurons.

Some of these principles were anticipated decades ago by investigators of the somatosensory system. For example, Johnson, Darian-Smith, and their colleagues attempted to relate the responses of "warm" and "cold" fibers in the peripheral nervous system to psychophysical sensitivity for temperature increments and decrements (Johnson et al., 1973, 1979). To our knowledge, they were the first to explore systematically the effect of pool size and correlated responsiveness on psychophysical performance. Johnson subsequently incorporated these and other themes into a formal theory relating neural activity to psychophysical discrimination (Johnson, 1980a,b). Unfortunately, these ideas could not be tested rigorously because electrophysiological recordings were never extended to suitable structures in the CNS. In the present study, recordings in the central visual system permitted us to 


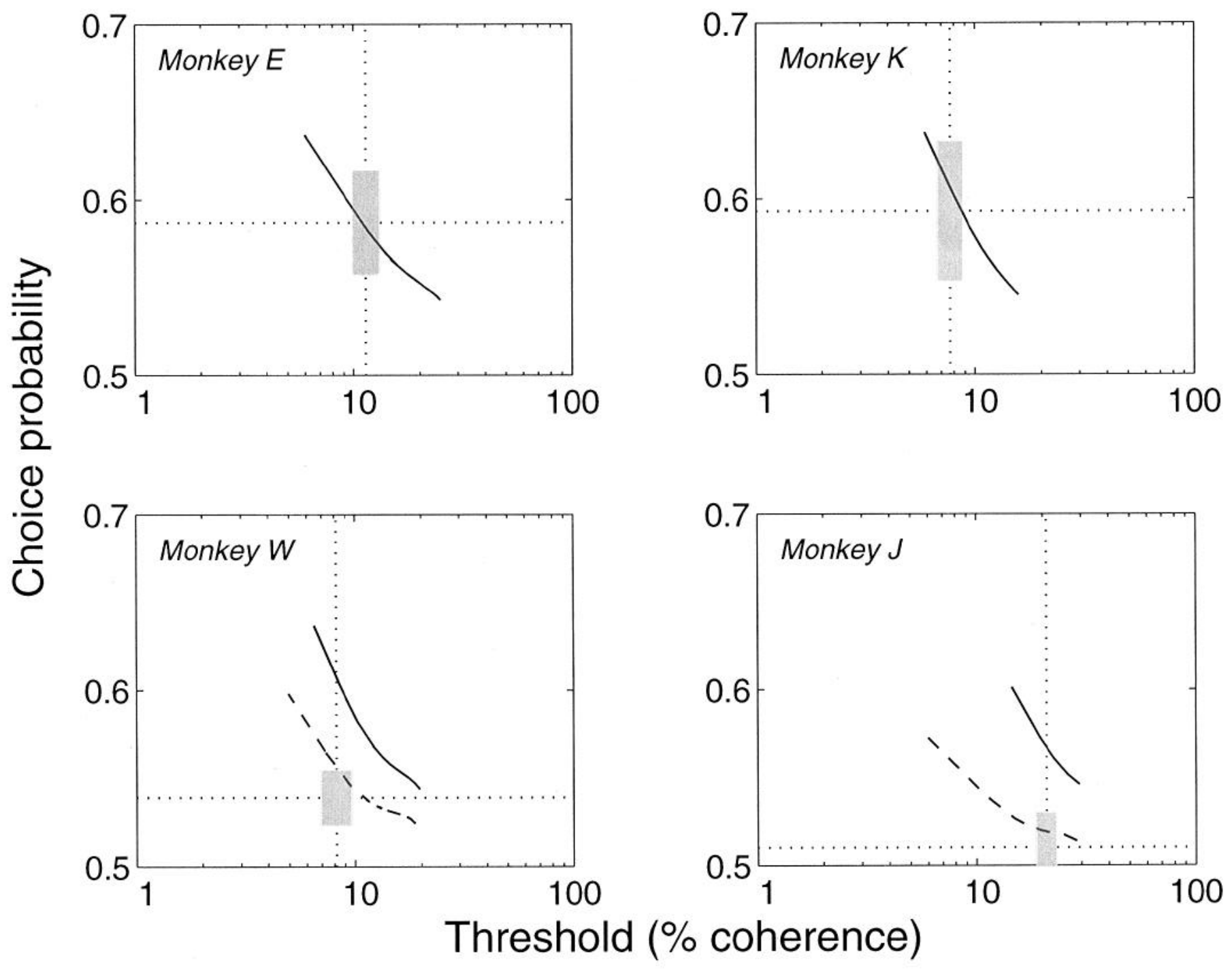

Figure 11. Model results for each of the four monkeys used in our experimental studies. Each simulation was based on neurons sampled from the monkey indicated in the top left corner of each graph. The pools also included less sensitive neurons than those recorded from each of the monkeys (mean unit threshold $=2.1-2.8$ times observed). Each curve shows the predicted Choice probability and psychophysical Threshold from the model as pooling noise increased from 0 to 2. Solid curves were obtained using model parameters identical to those in the "successful" model (curve CD in Fig. 6). Dashed lines (bottom panels) incorporated a lower mean correlation. For Monkey $W$, the mean correlation was 0.09 ; for Monkey J, the mean correlation was 0.05 . The dotted lines and shaded region indicate the mean \pm 2 SE for choice probability and threshold, for each monkey. Optimal simulation parameters for each monkey are summarized in Table 1.

measure the motion sensitivity, choice probability, and correlated responsiveness associated with sensory neurons underlying performance on the direction discrimination task. Together, these measures provide tighter experimental constraints on models linking physiology to psychophysics than have been available until now.

\section{Sources of error}

Our procedures incorporate enough assumptions, both implicit and explicit, that we have likely erred at some points. Let us consider four potential sources of error.

\section{Neuronal sensitivity}

It is possible that we have overestimated the sensitivity of single neurons in our sample by assuming that the brain can use the spikes generated over the entire $2 \mathrm{sec}$ stimulus epoch. If the average sensitivity of MT neurons were in fact lower than our estimates, psychophysical sensitivity could be modeled using neu- rons more like those in our data set-that is, without degrading the pools with less sensitive neurons. Supposing, for example, that the brain can only count spikes for $500 \mathrm{msec}$, the average sensitivity of neurons in our data set would be halved, obviating the need to incorporate less sensitive neurons in the pools.

We believe that this is unlikely for two reasons. First, psychophysical thresholds for our monkeys and for human observers improve as viewing times increase up to $2 \mathrm{sec}$ (Downing and Movshon, 1989; Britten et al., 1992), suggesting that spikes occurring at any time during the 2 sec epoch can influence the decision. Moreover, spikes counted from the final $500 \mathrm{msec}$ of a trial exhibit the same choice probability as spikes counted during the first 500 msec, again suggesting that no segment of the viewing interval is privileged in its impact on decisions (Celebrini and Newsome, 1994; Britten et al., 1996). Thus, it seems likely that our estimates of neuronal sensitivity are reasonably accurate and, therefore, that the pools of neurons influencing the judgments of the animals 
must contain neurons less sensitive than those we recorded. It is worth noting that revised estimates of neuronal sensitivity would not affect our conclusions concerning pooling noise. The amount of pooling noise incorporated in the best form of the model is determined solely by the need to reconcile experimental and simulated choice probabilities.

\section{Behavioral sensitivity}

Another potential source of error lies in our measurements of behavioral threshold. This has particular implications for our decision to include less sensitive neurons in the pools. Inspection of Figure 6 shows that if we formed the best model without the inclusion of suboptimally driven neurons, it would predict a value of behavioral threshold of $\sim 5 \%$, approximately half the value we observed (the point labeled $B$ ). If we had somehow consistently overestimated behavioral threshold, this might be the "true" value. We believe this is unlikely. The monkeys were trained on the motion discrimination task for many months before recordings were begun, and were performing quite stably then and thereafter. They routinely produced smooth and regular psychometric functions asymptoting near $100 \%$ performance; if they were not under complete behavioral control, we would expect the psychometric functions to asymptote at a lower value. It is nonetheless true that their behavioral thresholds varied from day to day in a way that we were unable to predict completely from such factors as the size, speed, and retinal locus of the motion display (Britten et al., 1992), and we cannot be entirely sure that some erroneously inflated thresholds were not included in our database. To the degree that this was true, our estimate of the proportion of less sensitive neurons to be included in the pools might be too high.

\section{Correlation}

Correlated noise among input neurons is a critical feature of the model, limiting the benefits of signal averaging and accounting for the choice probability associated with MT neurons. In our simulations, input neurons were typically assigned correlation coefficients varying randomly between 0 and 0.4 with a mean near 0.18 . These figures approximate the correlation coefficients measured in multiunit recordings from pairs of MT neurons (Zohary et al., 1994). Because these recordings were performed with a single electrode in combination with a spike sorter, we do not know the extent of correlated firing between neurons in different columns or between neurons at different locations in the same column. The observed values probably represent an upper bound on correlated firing, because adjacent neurons can safely be presumed to share common signals more than others (Braitenberg and Schüz, 1991; Stevens, 1994; Douglas et al., 1995). As illustrated in Figure 7, the hest form of our model can tolerate a range of average correlations between 0.1 and 0.3 .

Interestingly, the overall sensitivity of the pooled signal is limited by the average correlation within the pool, and the choice probability is determined by the average correlation of a neuron with other members of the pool (Zohary et al., 1994). The detailed connectivity is largely irrelevant. Thus, in extrapolating from measurements of correlation to the overall covariance structure of the pool, we require only that the these averages remain greater than 0.1 or so.

In all of our simulations, we assumed that neurons in the opposing direction pools are independent. Multiunit recordings from MT are consistent with this notion because neurons with disparate preferred directions were less well correlated than neu- rons with similar preferred directions (Zohary et al., 1994). We presume, however, that the opposing pools are drawn largely from columns with opposite preferred directions (consistent with microstimulation studies; see Salzman et al., 1992; Salzman and Newsome, 1994). In the event that noise in the opposing pools is positively correlated, comparison of activity in the two pools could negate their common noise, analogous to the action of a differential amplifier. Simulated thresholds should fall and choice probabilities should approach chance as expected for independent neural signals (Fig. 3, solid curves), creating an unbridgeable gap between simulated and experimental results. Thus, the model strongly predicts that correlations between cortical columns with opposed preferred directions will be substantially lower than correlations between columns with similar direction preferences. Pair recordings with multiple electrodes will be necessary to test this prediction.

\section{Pooling algebra}

We represented the pooled response as a simple average spike count. Clearly, this is an oversimplification; the pooled signal could be any scalar quantity that varies monotonically with the likelihood of one discriminandum relative to the other (Ihurstone, 1927, 1959; Green and Swets, 1966). Rather than pursuing a varicty of calculations, we chose to develop a set of heuristics based on a simple model.

Pooling arithmetic more complicated than averaging could lead to less efficient noise reduction. If, for example, we computed the harmonic mean or the geometric mean of the inputs, larger pool sizes might be required to reach asymptotic performance levels. The actual asymptotic values for threshold and choice probability are stable for a wide variety of calculations (for more examples, see Britten et al., 1992). Intuitively, these values reflect the common noise in the pool, and this noise remains after all of the uncorrelated noise is "averaged out" (see Appendices 2 and 3). Some computations, however, fail to achieve the same amount of noise reduction, even asymptotically. For example, the pooled average might be normalized by some other pooled quantity (Heeger, 1992). Such a computation would increase the variance of the pooled signal, thus reducing the need for pooling noise to reconcile experimental and simulated choice probabilities. In fact, we believe that this is a sound biological interpretation of the pooling noise in our model. The computations performed by any cortical circuit are likely to be more complex than a simple average, and the computed quantity is therefore likely to incorporate additional variance. Pooling noise in the model simply captures this additional variance (see Appendix 3).

\section{Alternative models}

The model architecture illustrated in Figure 2 is fairly general. A variety of implementations boils down to a pooling of MT signals followed by a comparison. Different assumptions about the information encoded in the spike train, the appropriate duration for the analysis, pooling algebra, and the parametric description of noise will lead to quantitative differences with the conclusions drawn here, but we would not anticipate substantial qualitative departures. We have considered, however, two alternatives in some detail: pooling models with small numbers of neurons and criterion-based models.

Most simulations in this paper incorporated asymptotically large pools of input neurons. The exquisite sensitivity of MT neurons led us, however, to explore models using small numbers of input neurons. We can produce a small-numbers pooling model 
that reconciles our experimental observations, but the parameters incorporated in the model make little biological sense. Approximately 10 neurons must be pooled to achieve the correct choice probabilities (Fig. 3C), but suboptimally driven neurons must be included in the pool to offset the gain in sensitivity that accrues from pooling among only 10 neurons $(\mathrm{Fig} .3 B)$; this is true even in the presence of pooling noise. Inclusion of such insensitive neurons in a small-numbers model scems dubious: the primary motivation for exploring small-numbers models is the extraordinary sensitivity of MT neurons, yet the pools must be degraded with insensitive neurons to make the model work. We believe that a small-numbers pooling model cannot work given plausible combinations of neural signals like those we recorded in MT.

Criterion-based models offer a more radical departure from the framework we have proposed. Here one assumes that a neurom signals the presence of motion toward a labeled direction when its response exceeds some criterion value. We have explored classes of models along these lines, varying parameters like criterion, pool size, and the method of conflict resolution when competing neurons exceed criterion. These efforts were largely unrewarding. Although we cannot exclude such models altogether, they generally predict erroneous values for choice probability or erroneous shapes of the psychometric function.

The best critcrion-based model we identified is a small-numbers scheme suggested to us by $\mathrm{H}$. Barlow. In this example, a pool of 5-6 neurons tuned for upward motion produces a perceptually effective signal when any two members of the pool yield responses $1 \mathrm{SD}$ above their background firing rates. For our 2-alternative forced-choice discrimination, a complementary pool of neurons must be incorporated that signals downward. This model works surprisingly well in some respects, but shares the general failures of this class of models, particularly in predicting the slopes of psychometric functions. For the interested reader, we present our implementation of this model in Appendix 4.

\section{The read-out problem: single neurons and populations}

In a sense, our modeling exercise is enveloped in irony. Our point of departure was our discovery that single MT neurons have sensitivity approximately equal to psychophysical sensitivity. After pooling the output of many such neurons, obtaining far superior sensitivity in the process, we then invoke two mechanisms to degrade this pooled signal to match psychophysical sensitivity. Why would the cortex pool neuronal signals only to discard the sensitivity gained? Why can signals from single MT neurons not guide performance?

This view is misleading simply because it is incomplete. A major goal of our sirnulations was to account for experimentally observed choice probabilities as well as perceptual thresholds. The need to reconcile both experimental results obliged us to accept large pool sizes, as is apparent from Figure 3. At a deeper level, however, we suspect that the signaling power of single neurons is compromised by lack of a sensitive mechanism to "read out" their signals. Impressive measurements of single-neuron sensitivity can be obtained by comparing precise spike counts using computers, but the critical issue is what counting and comparison operations can be accomplished with real cortical neurons. Imagine, for example, that the input pools in Figure 2 are populated with a single neuron each. These signals could be compared directly by a "decision neuron" that receives excitatory input from one sensory neuron and inhibitory input from the other. To effect this comparison with the reliability of our computer, however, connections from the signaling neurons to the comparison neuron would need to be highly privileged. Synaptic activity from other sources would have to be excluded lest the critical sensory signals become hopelessly confounded, leading to psychophysical performance substantially worse than that expected from computer calculations.

With few exceptions, privileged connections of this nature have not been documented in the cortex (Abeles, 1982; Stevens, 1994), and the brute facts of cortical microcircuitry render such notions questionable if not incredible (e.g., the typical cortical neuron receives several thousand synapses from a nearly equal number of neurons; see Peters, 1987; Braitenberg and Schüz, 1991; Douglas and Martin, 1991; Peters and Sethares, 1991; Shadlen and Newsome, 1994; Stevens, 1994; Douglas et al., 1995). Thus, the impressive signaling capabilities of single neurons appear ineffectual in the absence of a mechanism to evaluate and communicate these signals to subsequent processing stages. Our pooling model circumvents these difficulties through a redundant coding scheme. Because signals from multiple neurons are pooled at each stage of the pathway, noise is substantially reduced through averaging. The noise finally evident in behavior reflects limitations in noise reduction imposed by correlation.

This redundancy also offers important advantages for representing stimuli that fluctuate in time. If a temporally fluctuating motion pattern (like one of our stochastic dot displays) is coded by the spike rate of a single neuron having near-Poisson firing characteristics, a period equivalent to several interspike intervals is necessary to estimate the spike rate and, by extension, the amount of motion in the stimulus. If multiple neurons code the same stimulus, however, the mean spike rate can be estimated accurately in only one interspike interval, dramatically increasing the temporal resolution of the motion representation (Shadlen and Newsome, 1994).

The redundant coding scheme in our model is a specific example of a population code (Georgopoulos et al., 1986; Paradiso, 1988; Vogels and Orban, 1990; Zohary, 1992; Seung and Sompolinsy, 1993; Abbott, 1994), but as Barlow (1995) has pointed out, important distinctions must be made between classes of population codes. In any population code, different stimuli are represented by activity levels in distributed sets of neurons. The key issue is whether the set of active neurons is arbitrary, or whether each active neuron signals the presence of an important element in the input space. In the former scheme, exemplified by the ASCII code for alphanumeric characters, the state of any specific representational element gives very little information about the identity of the input. In the ASCII code, for example, a single bit is singularly uninformative concerning the identity of the represented character. In the alternative scheme, which Barlow terms a "direct representation," the activity of each representational element provides important information about the input. For example, each letter in the sequence S-T-I-M-U-L-U-S conveys information about the pronunciation of the word. Barlow argues that there are considerable advantages of direct representations in associative learning and in tolerance for errors.

The coding scheme embodied in our model is such a direct representation, allowing each neuron to influence psychophysical choices in accordance with its directional tuning. In this sense, our population code, like the vector averaging schemes of Gcorgopoulos and others, conforms to Barlow's essential insight that the response properties of an individual neuron provide critical information about its role in representing stimulus input or forming motor output. The precise size of the signaling pools seems less important than the principle that key features of the representa- 
tional code can be ascertained directly from the response of neurons sampled one at a time (Barlow, 1995).

\section{Concluding remarks}

Neurophysiologists studying sensory systems typically concentrate on maps and single-unit response properties as indicators of the representation of information in sensory structures. From our point of view, one of the most beneficial aspects of this modeling exercise is that we have been forced to think quantitatively about the impact on performance of such processes as correlated responsiveness, pooling, central noise, and decision mechanisms. These processes, although not often considered in detail by sensory physiologists, shape the output of a perceptual system just as profoundly as the initial stages of stimulus encoding.

\section{APPENDIX 1}

\section{Monte Carlo methods}

On each trial, we simulated a psychophysical decision by comparing responses from two pools of neurons. The neural response is represented as a random value, reflecting the number of action potentials discharged in the $2 \mathrm{sec}$ viewing period for a visual stimulus of given strength and direction. An overview of the model is provided in Figure 2 and sections describing our methods. IIere we supply additional details of the algorithm we used to simulate responses from neurons like those we recorded in area MT. We place particular emphasis on techniques for simulating weakly correlated responses from pools of neurons.

\section{Expected neural response}

The expected value for the response of the neuron was taken from our data set, but could be altered to simulate less sensitive neurons than those we encountered in our recordings. Thus, at the beginning of a simulation we picked $N$ neurons from our data set, randomly with replacement. Our model bases decisions on an opponent scheme involving two pools of neurons, with opposite preferred directions, and each neuron from the data set therefore played the role of two in the model. The measured response of the neuron to notion in its preferred direction provided the expected responses for a simulated cell in the "preferred" (up) pool, whereas the measured responses to motion in the null direction supplied the expected responses for a simulated unit in the "null" (down) pool. We used the same neurons in the two pools to guarantee that the expected response to $0 \%$ coherent dot motion was identical for the two pools, ensuring that simulated decisions were not biased in favor of either direction.

To model inclusion of suboptimally activated neurons, we associated a scaling factor, $b_{i}$, with each neuron selected for the two pools. The factor was chosen randomly from a range of values between 0 and 1 and used to scale the expected response of the neuron to motion of any given strength. The procedure had no effect on the response distribution to the $0 \%$ coherent dot display (random noise) but attenuated any gain or decrement in spike discharge associated with an increase in stimulus motion strength. If $\mu_{i}$ is the mean of the response of the $i$ th neuron to motion of strength, $c$, then the expected response of the model neuron is:

$$
m_{i}(c)=\mu_{i}(0)+b_{i}\left[\mu_{i}(c)-\mu_{i}(0)\right] \text {. }
$$

The expected response resembles the measured neural response when $b$ is near 1 . For small values of $b$, the simulated response was the same for all motion strengths, tending to the measured response to the $0 \%$ coherent motion display. Note that the simu- lated response to the $0 \%$ motion stimulus is unaffected by the scaling factor.

Scaling factors were chosen randomly from a beta distribution (Mood et al., 1963). The parameters of this distribution may be set so that $b$ is distributed uniformly on $[0,1]$, or concentrated near 1 , 0 , or any intermediate value. Using different parameters, we simulated pools of neurons of varying mixtures of sensitivity. We adopted this approach for its flexibility, but later learned that the shape of the distribution mattered little. As indicated in the text, the behavior of the model was essentially identical for all cases in which the average sensitivity of the neurons comprising the pools was the same, regardless of their distribution. It is perhaps worth noting, however, that the range of sensitivities derived from the successful model (Fig. 6, Table 1) was achieved using a uniform distribution of response scaling factors.

\section{Covariance}

We simulated a random response from each neuron in the pool for each of 500 trials for any one stimulus strength. The response was a random value generated as a normal deviate with mean:

$$
\left\langle x_{i}\right\rangle=m_{i}(c),
$$

and variance:

$$
\sigma_{i}^{2}=1.5\left\langle x_{i}\right\rangle=1.5 m_{i}(c)
$$

The angle brackets connote expectations of a random variable, which might be estimated by the mean of many trials. The choice of a Gaussian distribution is not critical to the model, but is convenient computationally. We chose the proportion of 1.5 after comparing our data with other estimates in the literature and attempting to discount long-term fluctuations in neural responsiveness, as discussed in the methods sections. Our goal was to generate random response values conforming to a desired set of expectations, and this variance rule, but to do so for $N$ neurons at a time in a manner ensuring correlation of desired magnitude. The method for generating these random responses is quite practical, and we believe it is more general than the one we have used previously (Britten et al., 1992). It represents a slight modification of a technique described by Földiák (1989).

For each trial, the responses from a pool of $N$ neurons can be represented as a vector, $\mathbf{x}$. We wish to generate these picks in a manner that realizes weak correlation between the elements of $\mathbf{x}$. That is, given $k$ trials, the correlation coefficient, $r_{i j}$, between the ordered pairs $x_{i k}$ and $x_{j k}$ should approximate some desired value:

$\left\langle r_{i j}\right\rangle=\frac{\operatorname{Cov}\left[x_{i}, x_{j}\right]}{\sqrt{\operatorname{Var}\left[x_{i}\right] \operatorname{Var}\left[x_{j}\right]}}=\frac{\left\langle x_{i} x_{j}\right\rangle-\left\langle x_{i}\right\rangle\left\langle x_{j}\right\rangle}{\sqrt{\left(\left\langle x_{i}^{2}\right\rangle-\left\langle x_{i}\right\rangle^{2}\right)\left(\left\langle x_{j}^{2}\right\rangle-\left\langle x_{j}\right\rangle^{2}\right)}}$,

For a pool of $N$ neurons, each neuron has some correlation with every other neuron in the pool. Thus, there are $N(N-1)$ pairwise correlations that comprise a correlation matrix. In most instances, we have chosen these pairwise correlations to be random, small, positive values, uniformly distributed on some interval (e.g., $0-0.4$ ). This task is tricky because many combinations of correlations are unrealizable. For example, if neurons $X_{1}$ and $X_{2}$ happen to be strongly correlated, then the correlation between $\mathrm{X}_{1}$ and a third neuron, $X_{3}$, constrains the possible correlations between $\mathrm{X}_{2}$ and $\mathrm{X}_{3}$. This means that we cannot generate correlation matrices by placing random values in a symmetric matrix.

Instead, we generate a random correlation matrix by approximating its matrix square root, $\mathbf{Q}$. The matrix, $\mathbf{Q}$, transforms a vector of independent normal deviates with zero mean and unit 
variance into a new vector of partially correlated values. The expected correlation matrix for these deviates is:

$$
\mathbf{C}=\mathbf{Q Q}^{\prime}
$$

The transformed deviates may be scaled and augmented to simulate the mean and variance associated with the response of any neuron.

This begs the question of how to generate $\mathbf{Q}$, the matrix square root of the random correlation matrix. Were the desired correlation values a constant, then the correlation matrix would appear as:

$$
\mathbf{C}=\mathbf{Q} \mathbf{Q}^{\prime}=\left(\begin{array}{ccc}
1 & r & \cdots \\
r & 1 & r \\
\vdots & r & \ddots
\end{array}\right)
$$

and the elements of $\mathbf{Q}$ would be:

$\mathbf{Q}=\left(\begin{array}{ccc}u & v & \cdots \\ v & u & v \\ \vdots & v & \ddots\end{array}\right)$

$u=\frac{1}{r \sqrt{N}} \sqrt{\frac{2}{N}+r-\frac{2 r}{N}-\frac{2}{N} \sqrt{(1-r)(1-r+r N)}}(1+\sqrt{(1-r)(1-r+r N)})$

$v=\frac{1}{\sqrt{N}} \sqrt{\frac{2}{N}+r-\frac{2 r}{N}-\frac{2}{N} \sqrt{(1-r)(1-r+r N)}}$

where $N$ is the number of elements. To generate the square root of a random correlation matrix, we use the mean correlation coefficient, $\bar{r}_{i j}$, to calculate the diagonal terms, and random picks for pairwise correlations coefficients $\left(r_{i j}=r_{j i}\right)$ to calculate the off-diagonal terms. Because this is just an approximation, it can be improved by forming $\mathbf{Q Q}^{\prime}$, and setting its diagonal terms to unity. The matrix square root of this adjusted correlation matrix must be computed and used to transform the independent normal deviates. In practice, the approximation works well, and we do not need to perform these last steps.

To form partially correlated responses from $N$ neurons, we generate a vector of independent, unit variance, zero mean, normal deviates, $z$, and multiply by the matrix square root of the desired correlation matrix:

$$
\mathbf{y}=\mathbf{Q z}
$$

Then the expected covariance of $\mathbf{y}$ is:

$$
\begin{array}{r}
\left\langle\mathbf{y} \mathbf{y}^{\prime}\right\rangle=\left\langle\mathbf{Q z}(\mathbf{Q z})^{\prime}\right\rangle=\left\langle\mathbf{Q z z} \mathbf{z}^{\prime} \mathbf{Q}^{\prime}\right\rangle=\left\langle\mathbf{Q} \mathbf{Q}^{\prime}\right\rangle=\langle\mathbf{C}\rangle \\
\text { since }\left\langle\mathbf{z z} \mathbf{z}^{\prime}\right\rangle=\mathbf{I} .
\end{array}
$$

Each of the deviates in $\mathbf{y}$ are then scaled according to a desired variance and augmented by their means. Because the variance is $\sim 1.5$ times the mean, the responses from $N$ neurons for a single trial are:

$$
\left\{x_{i}\right\}=\left\{m_{i}+\sigma_{i} y_{i}\right\}=\left\{m_{i}+\sqrt{1.5 m_{i} y_{i}}\right\}
$$

where $\left\{m_{i}\right\}$ is the vector of expected response values from each of the $N$ neurons, and $\left\{\sigma_{i}\right\}$ is their corresponding SD.

The operations defined by Equations A1.8 and A1.10 are repeated to simulate many trials to the same visual stimulus. Instead of a column vector of normal deviates, we begin with an $N$ by $M$ matrix, where $M$ is the number of trials. This yields a matrix of partially correlated deviates:

$$
\mathbf{Y}=\mathbf{Q Z}
$$

the rows of which are scaled and offset to achieve the desired set of expectations and variance (as in Eq. A1.10). The resulting matrix, $\mathbf{X}$, contains $M$ column vectors representing the responses from $N$ neurons on each of $M$ trials. The pooled responses are just the average of each of these column vectors. In principle, any function can be applied to the column to produce a pooled quantity. This quantity is compared with the pooled response from a competing set of neurons to simulate a decision.

Applying these steps, the responses from each neuron conform to a normal distribution. The method can be modified, however, to produce distributions of arbitrary shape, at the cost of computer processing time. We found no disadvantage to using normal deviates compared to more realistic distributions (e.g., scaled Poisson or lognormal). In general, it is reasonable to truncate deviates to positive response values. In our simulations, this had negligible impact because of the high background firing rate of most MT neurons. The actual set of correlations achieved in this manner approximates the desired values but is quite variable. We therefore calculated the actual correlation matrix from the simulated responses and averaged the off-diagonal terms. These are the values for correlation coefficients reported in the text.

In most of our simulations, we aimed to produce a random matrix of correlations, with desired mean. Because these pairwise correlations loosely reflect the connectivity between neurons in the pool, the model reflects many possible network architectures and is therefore quite general. In the end, however, it is the average correlation coefficient that determines the predicted sensitivity of the model. To predict the monkey's threshold, a random correlation matrix or a highly structured pattern of correlations produce the same predictions so long as they share the same mean $r$; this is not so for choice probability.

We discovered that a random set of correlations is unable to account for a systematic relationship between unit sensitivity and the magnitude of the choice probability in our data. To achieve this, we were forced to impose a relationship between unit sensitivity and its correlation strengths with other members of the pool. The correlation of each neuron with all of the other members of the pool is described by its multiple correlation coefficient:

$$
R_{i \cdot j \neq i}=\frac{\sqrt{\mathbf{s}_{(i)}^{\prime} \mathbf{C}_{22}^{-1} \mathbf{s}_{(i)}}}{\sqrt{s_{i i}}}
$$

where correlation matrix $\mathbf{C}$ has been partitioned as (Anderson, 1984):

$$
C_{i}=\left(\begin{array}{cc}
s_{i i} & \mathbf{s}_{(i)}^{\prime} \\
\mathbf{s}_{(i)} & \mathbf{C}_{22}
\end{array}\right)
$$

Almost equivalently, and perhaps more intuitively, we can use the avcrage of the desired corrclation cocfficients:

$$
\bar{r}_{i}=\frac{1}{N-1} \sum_{j \neq i}^{N} r_{i j}
$$

$R_{i}$ or $\bar{r}_{i}$ reflects the degree to which the $i$ th neuron is connected to the rest of the pool. A key insight is that this measure of correlation establishes the magnitude of the choice probability, because it reflects the degree to which the response of a single neuron covaries with the other members of the pool and, hence, the pool mean (see Appendix 2) (see also Zohary et al., 1994).

This strategy has certain limitations. Because we chose each pairwise correlation at random, the mean of each column of 
coefficients approaches the population mean of correlation coefficients for the matrix, $\bar{r}_{i j}$. Thus, each neuron tends to a common value for choice probability. This is important because it justifies our use of the mean choice probability to depict the performance of the model. Every neuron in the pool, regardless of its sensitivity, exhibits roughly the same choice probability. Unfortunately, this also implies that the model cannot account for the systematic variations we observed in choice probability for different neurons. To achieve different values of choice probability, we must impose different amounts of average correlation among the different neurons.

An example of such a correlation matrix was used to produce the results shown in Figure 11. For this set of connections, we generated a set of pairwise connections to the most sensitive neuron:

$$
r_{1 j}=r_{\min }+\frac{j}{N-1}\left(r_{\max }-r_{\text {min }}\right)
$$

In our simulation, these correlation coefficients ranged from 0.1 to 0.5. We allowed all of the remaining pairwise correlations to reflect the value expected because of common input with the best neuron:

$$
r_{i j, i>1, i \neq j}=r_{1 i} r_{1 j} .
$$

The correlation matrix is just the outer product of pairwise correlations to the best neuron, with the diagonal set to unity. The average correlation ranges from 0.3 for the most strongly connected neuron to $<0.01$. $\Lambda$ s above, the square root of the correlation matrix is used to transform normal deviates, which are then scaled to match the statistics of the neurons. The average pairwise correlation is dominated by the product terms and, therefore, tends to be rather small. For a pool size of 128 neurons, the average pairwise correlation was 0.11 .

To simulate the relationship between the choice probability and the threshold of the individual neuron, we arranged a correspondence between particular neurons and their correlations. More sensitive neurons were more strongly correlated with other neurons in the pool. We calculated the thresholds for each of neuron in the pool, and arranged the expectations, $\mathbf{m}$, so that the neurons with the lowest thresholds would end up with the largest average correlation. To do this, we generated a rank table for the $N$ average correlations (or multiple correlations) and used this to sort the desired values for the neurons, $\mathbf{m}$. As shown in Figure 10, the choice probabilities from such a model are heterogeneous and are indeed larger for more sensitive neurons.

The highly structured set of correlations used to produce Figure 10 is unrealistic. Ideally, we would construct quasirandom correlation matrices with a specified distribution of column averages or multiple correlation values $\left(R_{i}\right)$. This can be achieved iteratively, by transforming a small set of independent deviates by the square root of a structured correlation matrix, calculating the correlation matrix and using its square root. In practice, however, we found these steps ton time consuming to perform routinely.

\section{Decisions}

The matrix of responses, $\mathbf{Z}$, contains $N$ rows of response values for $M$ trials of identical motion. Each column is a vector of responses from the samc $N$ ncurons (with their associated scaling factors) to motion of a particular strength. The pooled signal is obtained by taking the column average:

$$
a_{j}=\frac{1}{N} \sum_{i=1}^{N} z_{i j}
$$

and using it to pick a random value from a normal distribution whose mean is $a_{j}$ and variance is $\nu_{a j}$, where $\nu$ is the pooling noise parameter (see Appendix 3). A random value of this sort is generated for each of the two pools ( $u p$ and down in Fig. 2) and compared in order to simulate the decision: the pool with the larger value wins.

\section{Choice probability}

The expected choice probability was calculated for each neuron using the $k$ trials for the $c=0 \%$ coherence stimulus strength. To do this, we compared the distribution of simulated responses when the decision was in the preferred direction of the neuron (up by the convention in Fig. 2) with the distribution associated with the opposite decision. The choice probability is the area under a receiver operating characteristic (ROC) curve formed from these two distributions, treating responses associated with up decisions as signal and down-decision responses as noise. Let $n$ be the number of preferred choices and $m$ be the number of null choices $(n+m=k$ ). Then, denoting $\theta$ as the list of responses associated with preferred choices, and denoting $\phi$ as the sorted list of null choice responses, the ROC curve is just the locus of points:

$$
\left\{\frac{N(\theta>\kappa)}{n}, \frac{N(\phi>\kappa)}{m}\right\}
$$

where $N()$ is the number of elements satisfying the condition in parentheses. The choice probability is the area under this ROC curve (Celebrini and Newsome, 1994; Britten et al., 1996).

An estimate for choice probability was obtained for each neuron in the pool for the $0 \%$ coherent motion stimulus. We calculated choice probabilities for nonzero motion strengths but found only minor effects of stimulus strength and direction.

\section{Psychometric function}

The simulated decisions were compiled into a standard psychometric function (PMF). The PMF represents the probability of a correct response as a function of stimulus strength (\% coherence). We obtained a PMF from 500 repetitions of 11 motion strengths using the same set of neurons, scaling factors, correlation matrix, and noise parameters. We fit this function with a cumulative Weibull distribution (see Eq. 1) to derive an estimate of psychophysical threshold and slope ( $\alpha$ and $\beta$ in Eq. 1). We computed geometric and arithmetic means for $\alpha$ and $\beta$, respectively, from several hundred PMFs, each time starting with a new set of neurons, scaling factors, and random correlation matrix.

\section{Neurometric function}

To characterize the sensitivity of single neurons to random dot motion, we determined the probability of correctly discriminating preferred from null direction motion based on the neuronal discharge. For each motion coherence, we constructed an ROC curve from the simulated neural response to motion in its preferred direction (signal) and null direction (noise) for a single motion coherence level. The area under the ROC curve corresponds to the probability that an ideal observer would correctly discriminate the direction of motion solely by comparing one response from each of these distributions. The neurometric function represents these derived probabilities as a function of stimulus strength. We fit this function with the cumulative Weibull density and derive the threshold $(\alpha)$. The sensitivity of a neuron is $\alpha^{-1}$. For simulations using response scaling factors, we compute the geometric mean of the threshold for each of the neurons in the pool and report this value normalized to the geometric mean 
of the same neurons, unattenuated. In some cases, the scaling procedure led to high thresholds that were poorly estimated by our fitting procedure. These scaled neurons were arbitrarily assigned a "threshold" of $100 \%$ coherence; because they were always a small minority of the simulated neuron pool, this had little effect on the simulations.

\section{APPENDIX 2}

\section{Signal-to-noise ratio in pools of mixed quality signals}

Here we develop a simple statistical analogy to guide intuitions about the properties of a pooled quantity computed from weakly correlated signals of varied sensitivity. We show that the signal-to-noise ratio (SNR) of the pooled average depends only on the average sensitivity of the pooled elements and their average pairwise correlation. The analysis suggests that, within limits, the exact composition of the pool affects the overall sensitivity relatively little. Second, this analysis provides an intuitive appreciation for our assertion that the choice probability reflects the average correlation of a neuron with other members of the pool.

\section{Signal-to-noise}

For simplicity, imagine that the neurons in our sample represent the best the brain has. Consider one such best signal, $X_{0}$, represented by a normally distributed random value with unity mean and variance. We take the signal to be a positive departure from zero. Hence, the SNR of this best signal is:

$$
\left\langle s /{ }_{N}\right\rangle_{x_{0}}=\frac{\left\langle X_{0}\right\rangle}{\sigma_{0}}=1
$$

In this and subsequent expressions, angle brackets denote expectation (or mean), and $\sigma$ denotes SD. We choose to make the SNR unity for convenience. This will establish a standard by which we may interpret additional signals of lesser quality.

What is the expected SNR of the average of $N$ weakly correlated signals, $X_{1} \ldots X_{N}$ ? It is the expectation of their average divided by their SE:

$$
\langle s / N\rangle=\frac{\frac{1}{N} \sum_{i=1}^{N} X_{i}}{\sqrt{\frac{1}{N^{2}} \sum_{i=1}^{N} \sum_{j=1}^{N} \operatorname{Cov}\left[X_{i}, X_{j}\right]}}
$$

The denominator is just the square root of the variance of a mean; we must consider the sum of covariances rather than the sum of variances because the signals are weakly correlated.

Suppose that each of the $N$ signals is a normally distributed random value with variance of 1 , but that their individual expectations range between 0 and 1 . In other words, the signals represent a mixture of qualities, with SNR between 0 and 1 . Let the mean value for this mixture be $m$. If $m$ is 1 , then we have $N$ signals that are all of the same quality as our best signal. If $m$ is $1 / 2$, then we have a mixture of signals that are, on average, half as sensitive as our best signal. This would arise, for example, if the expectations were themselves drawn from a uniform distribution between 0 and 1 .
The SNR of the average is just:

$$
\left\langle s / /_{N}\right\rangle=\frac{m}{\sqrt{\frac{1}{N^{2}} \sum_{i=1}^{N} \sum_{j=1}^{N} \operatorname{Cov}\left[X_{i}, X_{j}\right]}}
$$

$$
=\frac{m}{\sqrt{\frac{1}{N^{2}} \sum_{i=1}^{N} \sum_{j=1}^{N} r_{i j}, \sigma_{i} \sigma_{j}}}
$$

Because the SD of each of our signals is assumed to be unity, we can delete the subscripts from the $\sigma$ terms. The denominator is just the average of the values in the $N$ by $N$ covariance matrix defining the system of signals. This can be written as the sum of $N$ diagonal terms for variance, all equal to 1 , and $N(N-1)$ offdiagonal terms, $r_{i j}$. Notably, we can replace the individual pairwise correlations with their average, to obtain:

$$
\langle s / N\rangle=\frac{N m}{\sqrt{N+N(N-1) \bar{r}}}
$$

$$
\approx \frac{m}{\sqrt{r}} \text { for large } N
$$

The approximation for large $N$ works reasonably well even when $N$ is $50-100$. The approximation in Equation A2.4 is illuminating. It portrays the SNR of the average in units that are normalized to the $S / N$ of a single, best signal. For example, if the average correlation coefficient were 0.2 , then a mixture of signals with average SNR that is $45 \%$ that of the best source ( $m$ $=0.45$ ) would work about as well as the best source alone. Interestingly, even if we were to combine signals of the best quality $(m=1)$, we would achieve only a twofold improvement in SNR over any one source. As long as the signals are correlated, there is little to be gained from pooling the best signals and little to be lost in pooling signals of mixed quality. A crude mixture of sources that spans the range from completely insensitive to the most sensitive neuron will approximate the mixture that yields an SNR of the best neuron.

\section{Choice probability}

The analogy to choice probability is equally straightforward. Once again we consider a pooled quantity, like the mean of $N$ weakly correlated signals, $X_{1} \ldots X_{N}$. The choice probability is analogous to the correlation between any one signal, $X_{1}$, and the mean. The covariance between one signal and the mean can be written as:

$$
\begin{aligned}
\rho \sigma_{1} \sigma_{m} & =\rho \sigma \sqrt{\frac{1}{n^{2}} \sum_{i=1}^{N} \sum_{j=1}^{N} \operatorname{Cov}\left[X_{i}, X_{j}\right]} \\
& =\frac{\rho \sigma}{N} \sqrt{N \sigma^{2}+N(N-1) \bar{r} \sigma^{2}}
\end{aligned}
$$

where $\rho$ is the correlation analogous to the choice probability, $\sigma_{m}$ is the SEM, and $\bar{r}$ is the average correlation coefficient between any pair of signals. The left side of Equation A2.5 can 
also be written in terms of products and their expectations:

$$
\begin{aligned}
\rho \sigma_{1} \sigma_{m} & =\operatorname{Cov}\left[X_{1}, \frac{1}{N} \sum_{i=1}^{N} X_{i}\right] \\
& =\left\langle X_{1} \sum_{i=1}^{N} \frac{X_{i}}{N}\right\rangle-\left\langle X_{1}\right\rangle\left\langle\sum_{i=1}^{N} \frac{X_{i}}{N}\right\rangle \\
& =\frac{1}{N}\left(\left\langle X_{1}^{2}\right\rangle+\sum_{i=2}^{N}\left\langle X_{1} X_{i}\right\rangle-N\langle X\rangle^{2}\right) \\
& =\frac{1}{N}\left(\sigma^{2}+\sum_{i=2}^{N} \operatorname{Cov}\left[X_{1}, X_{i}\right]\right) \\
& =\frac{1}{N}\left(\sigma^{2}+(N-1) \bar{r}_{1} \sigma^{2}\right)
\end{aligned}
$$

where $\bar{r}_{1}$ is the average correlation coefficient between $X_{1}$ and each of the other signals, $X_{2} \ldots X_{N}$. Solving for $\rho$ in Equations 2.5 and 2.6, we obtain (Zohary et al., 1994):

$$
\begin{aligned}
\rho & =\frac{1+(N-1) \bar{r}_{1}}{\sqrt{N+N(N-1) \bar{r}_{1}}} \\
& \approx \sqrt{\bar{r}_{1}} \text { for large } N
\end{aligned}
$$

In words, the correlation between one signal and the mean to which it contributes depends on the average correlation of that signal with the other signals that comprise the mean. The quality of the signals does not affect this correlation unless we postulate a connection between correlation and sensitivity. For the simulations in Figure 4, we used random pairwise correlations averaging 0.18 . So inclusion of relatively poorly driven neurons did not affect the choice probability. In Figure 10, the more sensitive neurons were more strongly correlated with other members of the pool, and these neurons exhibited larger choice probabilities.

\section{APPENDIX 3}

\section{Pooling noise}

To simulate pooling noise, we added variability to the two pooled responses before comparing them to render a decision. On each simulated trial, we added a zero-mean, normally distributed random value to each of the pooled means. The variance of the noise distribution was proportional to the pooled mean itself and therefore differed from trial to trial and between pools. We parameterized the pooling noise in our model by a constant of proportionality: variance divided by the pooled mean, or variance-tomean ratio (VMR). Our simulations worked best when this ratio was quite small $(0.2-0.4)$. Here we try to develop an intuition for this quantity. We first consider the range of values for pooling noise that makes sense biologically. We then develop a more general heuristic for thinking about pooling noise. Interestingly, this heuristic, based on general experimental observations, leads one to expect pooling noise values very similar to those derived in this paper from simulations of a specific set of neuronal and psychophysical data.

Pooling noise represents all sources of variability that affect the representation of the pooled average and its transmission to neural processes that render a decision. At the lower end of the range of plausible values, psychophysical performance and neuronal choice probability might be simulated adequately without any addition of pooling noise. This result would imply that the variability in psychophysical performance can be accounted for completely by response variability measured in MT. Higher-order neuroms that pool the outputs of MT would achieve the same degree of noise reduction as our simulations by computing the average of the MT responses, adding no additional noise of their own. An upper bound for pooling noise is less certain because many stages of neural processing might intervene between MT and formation of the psychophysical decision. Nevertheless, it seems reasonable to expect these processes to do better than a single neuron at representing the pooled quantities. The VMR for neurons in several cortical areas is reported to be on the order of 1.5. $\Lambda$ value for pooling noise exceeding 1.5 would imply that the brain cannot represent the pooled motion signals as well as a single neuron.

A general framework for interpreting pooling noise would require an understanding of the actual physiological sources of variability in neuronal responsiveness. However, a simple heuristic lends some insight. Consider the response from a hypothetical neuron that computes the pooled average-one of unany such neurons, presumably. We will show that such a neuron would possess the same amount of variability as other cortical neurons, provided we incorporate pooling noise in our estimate.

The variance of the spike count of the pooling neuron might be viewed as the sum of a conditional variance and the variance of the quantity to be represented:

$$
E[\operatorname{Var}[Y]]=E\left[\operatorname{Var}\left[Y \mid X_{1} \ldots X_{N}\right]\right]+\operatorname{Var}\left[E\left[Y \mid X_{1} \ldots X_{N}\right]\right]
$$

In this expression, $X_{1} \ldots X_{N}$ are random variables that represent the responses from MT, say, the spike counts from neurons in the $u p$ pool. $Y$ is the response of our hypothetical pooling neuron; it represents the mean of $X_{1} \ldots X_{N}$; hence, its expected value is $\bar{X}$. $E$ [] stands for the expectation of the quantity within the brackets, and $Y \mid X_{1} \ldots X_{N}$ should be read, " $Y$, given a fixed set of inputs." Equation A3.1 expresses the expected variance of the response of the pooling neuron as the sum of two terms. The first term of the sum is the conditional variance: given the same set of responses from MT, the pooling neuron would nevertheless generate a different response on each trial, and this is the expected variance. This term is presumably related to processes governing synaptic integration and spike generation-that is, the process that makes the interspike interval irregular (Shadlen and Newsome, 1994). For example, if the neuron is modeled as a Poisson point process, the conditional variance should equal its expectation. In other words, even if the pooled mean, $\bar{X}$, were 100 spikes on every trial, $Y$ would vary with $($ mean $=$ variance $=100)$. In reality, the interspike interval may be more regular than a Poisson point process. The coefficient of variation $\left(C_{\nu}\right)$ of the interspike interval may be closer to 0.8 (Softky and Koch, 1993). This would imply that the conditional variance of the spike count is $\sim 0.64-1.0$ (i.e., $C_{\text {v }}^{2}$ ) times the mean (see Smith, 1959).

The right-hand term in Fquation A3.1 is the variance of the expectation itself. This term reflects the variability in the quantity that the pooling neuron is supposed to calculate in the first place. Ideally, this is the pooled mean spike count from MT (i.e., $X$ ). Recall that pooling does not produce a zero variance signal. Weak correlation among MT neurons leads to an asymptotic variance reflecting the common noise. Were the pooling operation a simple 
mean, the variance would be $1.5 \bar{r}_{i j} \bar{X}$, the average variance times the average correlation (Zohary et al., 1994). Accepting the average correlation in $\mathrm{MT}$ as $\sim 0.2$, we get a VMR of 0.3 for the computed mean. By adding the two terms on the right side of Equation A3.1, we are imagining that the pooling neuron computes a value that is variable (the variance of the conditional expectation) and then compounds this variability with its own spiking irregularity (the conditional variance). Thus, we would anticipate a total variance of $0.94-1.3$ times the mean.

There is nothing intrinsically wrong with these values. However, it is reasonable to expect that pooling neurons behave statistically like MT neurons - that is, they too should exhibit a VMR of 1.5. To achieve this amount of variability, we must boost the variance by $0.2-0.56$ times the mean, a value that is remarkably consistent with the amount of pooling noise added to the model to satisfy experimentally derived constraints (Figs. 6, 7). In terms of Equation A3.1, this is variance that belongs lumped in the right-hand expression (variance of the conditional expectation). Presumably, it corrects our oversimplifying assumption that the pooling is achieved as an average.

Put another way, the requirement of the model for pooling noise implies a similarity between neurons at the pooling slage and those in area MT. The variance of the pooled mean combined with pooling noise and the neuron's intrinsic variability sum to a VMR of $\sim 1.5$. Thus, a putative pooling neuron is no more or less noisy than an average MT neuron, implying that noise has achieved a steady state by the level of MT. This is an attractive notion because it helps us understand how simple sensory signals can be propagated accurately through successive stages in a complex neural pathway. Each stage involves additional circuitry (to achieve novel computational capabilities, presumably) which, in turn, adds some noise to the signal ("pooling noise"). This noise added at each stage is rather precisely balanced, however, by the noise reduction achieved through pooling. Thus, simple signals can be transmitted faithfully through an indefinite number of higher stages before ultimately reaching decisional and premotor centers.

In our original modeling exercise, pooling noise was a somewhat arbitrary quantity needed to reconcile experimental and simulated data. In this more general conception, however, pooling noise is an inevitable concomitant of the complex circuitry needed for computational power, and it is finely balanced at each stage of processing by noise reduction achieved through averaging signals across a pool of neurons. Interestingly, quantitative estimates of pooling noise derived through the two approaches agree well.

\section{APPENDIX 4}

\section{Example of an alternative model architecture}

Here we consider a criterion-based model for the monkey's psychophysical behavior. The model we have considered thus far works on the principle of maximum likelihood: it chooses the most likely direction of motion, given the neural discharge. A criterionbased model works by detecting motion when some neuron or set of neurons exceeds a criterion response. Because this notion uses a fundamentally different set of assumptions, we describe it in detail. Moreover, it represents our most successful attempt to model the monkey's behavior on the basis of a small number of neurons.

Rather than pooling responses from MT and passing the result to a decision, this model assumes that each MT neuron signals the presence of a specific feature of visual motion. Consistent with the notion of feature detectors (Lettvin et al., 1959; Martin, 1994) and the "neuron doctrine" of perception (Barlow, 1972, 1995), the neural response leads more or less directly to the impression of motion. In our task, the monkey is required to choose between one of two directions. We must therefore construct a model that converts feature detection to psychophysical decisions.

Suppose that just a few neurons are feature detectors optimally suited to detect motion in our display. That is, given the location of the random dot motion stimulus, its size, speed, and disparity, there are only a small number of feature detectors that the brain uses to support its judgment of direction. Suppose that this number is 5: on each trial, each of 5 neurons responds to the stimulus motion in the manner observed in our experiments. Stronger motion (higher dot coherence) leads to a larger response. The simulated response is quite variable, so that multiple trials with identical stimuli lead to a distribution of responses with variance 1.5 times the mean. If any 2 neurons (of the 5) exceed some criterion, then the monkey detects motion in the direction of this neuron. We will qualify the word detect in a moment; it is not synonymous with decide. The criterion is the main free parameter in the model. Assume, for the moment, that it is $1 \mathrm{SD}$ above the response to the $0 \%$ coherent motion display. For example, an upward-preferring neuron that discharges 40 spikes, on average, to the $0 \%$ coherent display detects upward motion whenever its response exceeds $40+\sqrt{(1.5)(40)} \sim 48$ spikes.

Of course, for every 5 neurons selective for motion in the upward direction, there are also neurons selective for the opposite direction. Again, if any 2 of 5 neurons exceed criterion, then the monkey is said to detect downward motion. Notice that it is possible to detect motion in opposite directions, simultaneously, particularly with weak motion strengths. It is also possible that neither pool of neurons achicves critcrion for a detcction. Wc analyzed several decision rules to handle these contingencies. The rule that worked best was to break ties resulting from neither side achieving detection with a coin toss (i.e., a guess) and to break ties resulting from both pools achieving detection by deciding in favor of the pool with the larger number of votes $(>2)$; if this did not produce a winner, we resorted to a coin toss. Notice that, unlike the model considered elsewhere in this paper, neural signals are not combined to improve the SNR of the stimulus representation. Here they combine as independent detectors, through probability summation (Watson, 1979; Graham, 1989).

The free parameters in the model are as follows: (1) the sensitivity of the neurons; (2) the number of neurons in each detecting unit; (3) any weak correlation among their responses; (4) the criterion response for a neuron to detect motion; and (5) the number of neurons exceeding criterion required for a detection. We can simplify this by considering only neurons that were recorded in our data set. It makes little sense to imagine that the monkey bases its decision on a few neurons, but chooses relatively insensitive ones. In a similar vein; we assume that the few neurons the monkey consults respond essentially independently (weak correlation is much less important with small numbers of neurons). We show results for the model that requires 2 neurons to exceed criterion because this turned out to be the best. This leaves two parameters for analysis: the number of neurons and the criterion response.

The results are shown in Figure 12. The effect of criterion and neuron number are shown for three model predictions: psychophysical threshold, the slope of the PMF, and the choice probability for the individual neurons. The plotted values are averaged from 200 simulations using neurons randomly sampled from monkeys $\mathrm{E}$ and $\mathrm{K}$. The model predicts psychophysical thresholds equal 

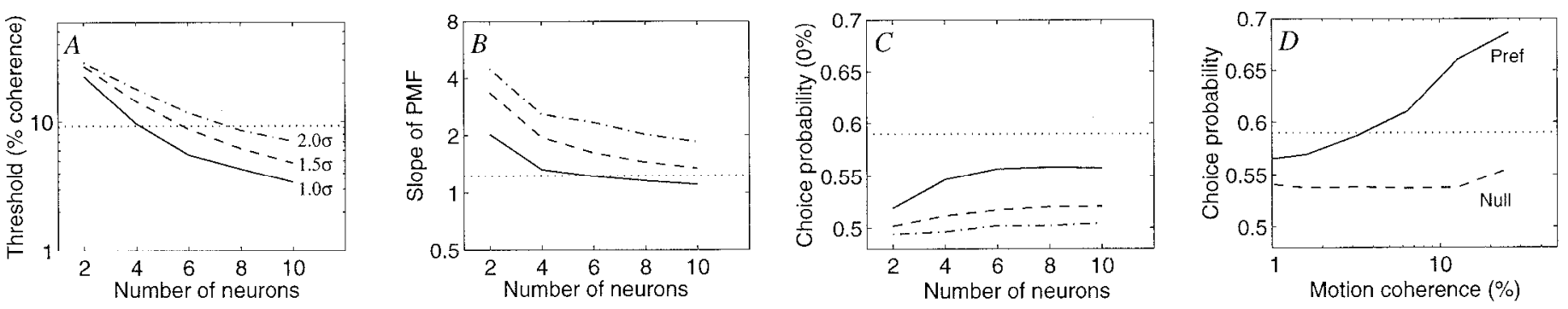

Figure 12. These plots summarize the predictions from our most successful model using small numbers of neurons. A small group of neurons was taken to detect motion in its preferred direction if any two exceeded a criterion response. A second group of neurons detected motion in the opposite direction using the same rule as the first. Ties uccurred when both groups signaled a detection, or when neither did. Ties were resolved by a coin toss. $A$, Predicted Threshold for the model is plotted as a function of group size. Solid curve, Criterion $=1 \mathrm{SD} ;$ Dashed curve, criterion $=1.5 \mathrm{SD} ;$ Dot-dash curve, criterion $=2 \mathrm{SD}$. The horizontal dotted line indicates the observed mean threshold for monkeys $\mathrm{E}$ and $\mathrm{K}$. $B$, The Slope of PMF plotted as a function of group size. Curves depict the same criterion values as in $A$. Conditions that foster ties tended to produce steep psychometric functions. 'l'he horizontal dotted line indicates the observed mean slope for monkeys $\mathrm{E}$ and $\mathrm{K}$. C, Choice probability for individual neurons is plotted as a function of group size. Three curves represent different criteria as in $A$. Choice probability was computed for the $0 \%$ coherent motion stimulus. The dotted line shows the value observed in monkeys $\mathrm{E}$ and K. D, Influence of stimulus strength and direction on Choice probability. Average choice probability for the $N=5$ model with criterion $=1 \mathrm{SD}$ is plotted as a function of stimulus strength. For the solid curve, motion was in the preferred direction of the neuron; for the dashed curve, motion was in the null direction of the neuron.

to those observed in our monkeys when any 2 of $4-8$ neurons provide the detection (Fig. 12A). Threshold falls with increasing numbers of neurons. Not surprisingly, threshold is higher with more stringent detection criteria (dashed curves). The model predicts a steeper PMF than we observed in our experiments (Fig. $12 B$ ). The value for slope here is $\beta$ in the best-fitting cumulative Weibull function (Eq. 1). In our data, the mean value was 1.22, as indicated by the horizontal dotted line. The criterion-based model tends to predict steeper slopes, especially for lower values of $N$ and higher criteria.

The model predicts a weak choice probability for $0 \%$ coherent motion (Fig. 12C). Although none of the curves matches the data perfectly, the low criterion model (solid cune) nearly approximates the observed mean value for monkeys $\mathrm{E}$ and $\mathrm{K}$, using 5-8 neurons. The choice probability is small because many decisions result from guesses. In contrast, the model considered throughout the body of the paper rarely resorted to guessing; consequently, the choice probability was larger, especially when small numbers of neurons governed the decision (compare Fig. $3 C$ ). It is reasonable, however, to wonder whether the average choice probability is what a "small-numbers" model should predict. The more sensitive neurons in our data had larger choice probabilities, and it is presumably these neurons that would be used in such a model. A more serious problem arises when we consider motion strengths other than $0 \%$ coherence (Fig. 12D). The choice probability rises precipitously for stronger motion in the preferred direction, because fewer decisions result from guesses. This trend is not supported by our data (Britten et al., 1996), nor is it predicted by the model described in the main text.

This last reservation aside, the model does reasonably well at predicting both psychophysical performance and choice probability. The model is quite delicate, in that there is a rather narrow range of parameters that produce reasonable values for threshold and slope of the psychometric function. For example, the results shown in Figure 12 depend critically on the particular choice of neurons we have incorporated: 2-6 neurons chosen randomly from our sample. If the monkey is to consult only 5 neurons, why would it choose only those of average sensitivity, or worse yet, a mixture of sensitivities spanning two orders of magnitude? If we incorporate only neurons of above average sensitivity, then we can no longer account for the psychophysical results, because the model predicts sensitivity to be too high. We cannot fix this by raising the detection criterion or by increasing the number of neurons required to exceed criterion, because these maneuvers also steepen the slope of the psychometric function unacceptably. Intuitively, the model operates in two modes, one in which there is a clear winner and one in which the decisions are attributed to guesses. Raising the criterion extends the range of stimulus strengths over which judgments arise through guesses, driving the performance toward chance for weak stimuli while negligibly affecting performance on stronger stimuli. This means that a change in threshold is accompanied by a change in the slope of the PMF, often to unacceptable values.

\section{REFERENCES}

Abbott LF (1994) Decoding neuronal firing and modelling neural networks. Q Rev Biophys 27:291-331.

Abeles M (1982) Local cortical circuits. Berlin: Springer.

Allman JM, Kaas JH (1971) A representation of the visual field in the caudal third of the middle temporal gyrus of the owl monkey (Aotus trivirgatus). Brain Res 31:85-105.

Anderson TW (1984) An introduction to multivariate statistical analysis. New York: Wiley.

Barlow HB (1972) Single units and sensation: a neuron doctrine for perceptual psychology? Perception 1:371-394.

Barlow HB (1995) The neuron doctrine in perception. In: The cognitive neurosciences (Gazzaniga M, ed), pp 415-435. Cambridge: MIT.

Bradley A, Skottun BC, Ohzawa I, Sclar G, Freeman RD (1987) Visual orientation and spatial frequency discrimination: a comparison of single cells and behavior. J Neurophysiol 57:755-772.

Braitenberg V, Schüz A (1991) Anatomy of the cortex: statistics and geometry. Berlin: Springer.

Britten KH, Shadlen MN, Newsome WT, Movshon JA (1992) The analysis of visual motion: a comparison of neuronal and psychophysical performance. J Neurosci 12:4745-4765.

Britten KH, Shadlen MN, Newsome WT, Movshon JA (1993) Responses of neurons in macaque MT to stochastic motion signals. Vis Neurosci 10:1157-1169.

Britten KH, Newsome WT, Shadlen MN, Celebrini S, Movshon JA (1996) A relationship between behavioral choice and the visual responses of neurons in macaque MT. Vis Neurosci 13:87-100.

Celebrini S, Newsome WT (1994) Neuronal and psychophysical sensitivity to motion signals in extrastriate area MST of the macaque monkey. J Neurosci 14:4109-4124.

Dean AF (1981) The variability of discharge of simple cells in cat striate cortex. Exp Brain Res 44:437-440.

Douglas RH, Martin KAC (1991) A functional microcircuit for cat visual cortex. J Physiol (Lond) 440:735-769. 
Douglas RJ, Koch C, Mahowald M, Martin KAC, Suarez HH (1995) Recurrent excitation in neocortical circuits. Science 269:981-985.

Downing CJ, Movshon JA (1989) Spatial and temporal summation in the detection of motion in stochastic random dot displays. Invest Ophthalmol Vis Sci 30:72.

Dubner R, Zeki SM (1971) Response properties and receptive fields of cells in an anatomically defined region of the superior temporal sulcus. Brain Res 35:528-532.

Efron B, Tibshirani RJ (1993) An introduction to the bootstrap (Cox D, Hinkley D, Reid N, Rubin D, Silverman B, eds). New York: Chapman \& Hall.

Földiák P (1989) Adaptive network for optimal linear feature extraction. In: IEEE/INNS international joint conference on neural networks, Vol 1, pp 401-405. Washington, D.C.: IEEE.

Gawne TJ, Richmond BJ (1993) How independent are the messages carried by adjacent inferior temporal cortical neurons? J Neurosci 13:2758-2771.

Georgopoulos AP, Schartz AB, Kettner RE (1986) Neuronal population coding of movement direction. Science 233:1416-1419.

Graham NVS (1989) Visual pattern analyzers. New York: Oxford UP.

Green DM, Swets JA (1966) Signal detection theory and psychophysics. New York: Wiley.

Heeger DJ (1992) Normalization of cell responses in cat striate cortex. Vis Neurosci 9:181-198.

Johnson KO (1980a) Sensory discrimination: decision process. J Neurophysiol 43:1771-1792.

Johnson KO (1980b) Sensory discrimination: neural processes preceding discrimination decision. J Neurophysiol 43:1793-1815.

Johnson KO, Darian-Smith I, LaMotte C (1973) Peripheral neural determinants of temperature discrimination in man: a correlative study of responses to cooling skin. J Neurophysiol 36:347-370.

Johnson KO, Darian-Smith I, LaMotte C, Johnson B, Oldfield S (1979) Coding of incremental changes in skin temperature by a population of warm fibers in the monkey: correlation with intensity discrimination with man. J Neurophysiol 42:1332-1353.

Lettvin JY, Maturana HR, McCulloch WS, Pitts WH (1959) What the frog's eye tells the frog's brain. Proc Inst Rad Eng 47:1940-1951.

Martin KAC (1994) A brief history of the "feature detector." Cereb Cortex 4:1-7.

Mood AM, Grabill FA, Boes DC (1963) Introduction to the theory of statistics. New York: McGraw-Hill.

Mountcastle VB, Talbot WH, Sakata H, Hyvarinen J (1969) Cortical neuronal mechanisms in flutter-vibration studied in unanesthetized monkeys: neuronal periodicity and frequency discrimination. J Neurophysiol 32:452-484.

Mountcastle VB, LaMotte RH, Carli G (1972) Detection thresholds for vibratory stimuli in humans and monkeys: comparison with threshold events in mechanoreceptive first order afferent nerve fibers innervating monkey hands (Abstr). J Neurophysiol 35:122A.

Nachmias J (1980) On the psychometric function for contrast detection. Vision Res 21:215-223.

Newsome WT, Britten KH, Movshon JA (1989) Neuronal correlates of a perceptual decision. Nature 341:52-54.

Newsome WT, Shadlen MN, Zohary E, Britten KH, Movshon JA (1995) Visual motion: linking neuronal activity to psychophysical performance. In: The cognitive neurosciences (Gazzaniga M, ed), pp 401-414. Cambridge: MIT.

Paradiso MA (1988) A theory for the use of visual orientation information which exploits the columnar structure of striate cortex. Biol Cybern 58:35-49.
Peters A (1987) Number of neurons and synapses in primary visual cortex. In: Cerebral cortex. Further aspects of cortical function, including hippocampus, Vol 6 (Jones E, Peters A, eds), pp 267-294. New York: Plenum.

Peters A, Sethares C (1991) Organization of pyramidal neurons in area 17 of monkey visual cortex. J Comp Neurol 306:1-23.

Quick RF (1974) A vector magnitude model of contrast detection. Kybernetik 16:65-67.

Salzman CD, Newsome WT (1994) Neural mechanisms for forming a perceptual decision. 264:231-237.

Salzman CD, Britten KH, Newsome WT (1990) Cortical microstimulation influences perceptual judgments of motion direction. Nature 346:174-177.

Salzman CD, Murasugi CM, Britten KH, Newsome WT (1992) Microstimulation in visual area MT: effects on direction discrimination performance. J Neurosci 12:2331-2355.

Seung HS, Sompolinsy H (1993) Simple models for reading neuronal population codes. Proc Natl Acad Sci USA 90:10749-10753.

Shadlen MN, Newsome WT (1994) Noise, neural codes and cortical organization. Curr Opin Neurobiol 4:569-579.

Smith WL (1959) On the cumulants of renewal processes. Biometrika 46:1-29.

Snowden RJ, Treue S, Andersen RA (1992) The response of neurons in areas $\mathrm{V} 1$ and $\mathrm{MT}$ of the alert rhesus monkey to moving random dot patterns. Exp Brain Res 88:389-400.

Softky WR, Koch C (1993) The highly irregular firing of cortical cells is inconsistent with temporal integration of random EPSPs. $\mathrm{J}$ Neurosci $13: 334-350$.

Stcvens CF (1994) What form should a cortical theory take? In: Largescale neuronal theories of the brain (Koch C, Davis J, eds), pp 239-256. Cambridge: MIT.

Thurstone LL (1927) A law of comparative judgement. Psychol Rev 34:273-286.

Thurstone LL (1959) The measurement of values. Chicago: University of Chicago.

Tolhurst DJ, Movshon JA, Dean AF (1983) The statistical reliability of signals in single neurons in cat and monkey visual cortex. Vision Res 23:775-785.

Van Essen DC, Maunsell JHR, Bixby JL (1981) The middle temporal visual area in the macaque: myeloarchitecture, connections, functional properties and topographic representation. $J$ Comp Neurol 199:293-326.

van Kan PLE, Scobcy RP, Gabor AJ (1985) Rcsponsc covariance in cat visual cortex. Exp Brain Res 985:559-563.

Vogels R, Orban GA (1990) How well do response changes of striate neurons signal differences in orientation: a study in the discriminating monkey. J Neurosci 10:3543-3558.

Vogels R, Spileers W, Orban GA (1989) The response variability of striate cortical neurons in the behaving monkey. Exp Brain Res $77: 432-436$.

Watson AB (1979) Probability summation over time. Vision Res 19:515-522.

Werner G, Mountcastle VB (1963) The variability of central neural activity in a sensory system, and its implications for the central reflection of sensory events. J Neurophysiol 26:958-977.

Zohary E (1992) Population coding of visual stimuli by cortical neurons tuncd to more than onc dimension. Biol Cybcrn 66:265-272.

Zohary E, Shadlen MN, Newsome WT (1994) Correlated neuronal discharge rate and its implications for psychophysical performance. Nature $370: 140-143$ 